\title{
Archaeonautica
}

Archaeonautica

L'archéologie maritime et navale de la préhistoire à

l'époque contemporaine

$20 \mid 2018$

De re navali : Pérégrinations nautiques entre

Méditerranée et océan Indien

\section{Capo Sagro 2 : une épave romaine à chargement de lingots d'étain à 500 mètres de profondeur}

Capo Sagro $2:$ a roman wreck loading tin ingots 500 meters depth

Franca Cibecchini, Christian Rico et Pierre Poveda

\section{OpenEdition}

Édition électronique

URL : http://journals.openedition.org/archaeonautica/438

DOI : $10.4000 /$ archaeonautica.438

ISSN : 2117-6973

Éditeur

CNRS Éditions

Édition imprimée

Date de publication : 6 décembre 2018

Pagination : 67-87

ISBN : 978-2-271-12263-6

ISSN : 0154-1854

Référence électronique

Franca Cibecchini, Christian Rico et Pierre Poveda, «Capo Sagro 2 : une épave romaine à chargement de lingots d'étain à 500 mètres de profondeur », Archaeonautica [En ligne], 20 | 2018, mis en ligne le 30 avril 2020, consulté le 30 avril 2020. URL : http://journals.openedition.org/archaeonautica/438 ; DOI : https://doi.org/10.4000/archaeonautica.438 


\title{
CaPO SaGRo 2 : une ÉPAVE ROMAINE À CHARGEMENT DE LINGOTS D'ÉTAIN À 500 MÈTRES DE PROFONDEUR
}

\author{
Franca CibeCChINI, Christian Rico, Pierre PovedA
}

\begin{abstract}
Résumé
L'épave Capo Sagro 2 gît par près de 500 m de fond au large du cap du même nom à quelques miles au nord-est des côtes corses. Découverte de façon fortuite, les différentes plongées robotisées de vérification ont très vite montré le caractère inhabituel du chargement composé en très grande majorité de lingots d'étain.

Le relevé photogrammétrique du tumulus a mis en évidence la relative homogénéité de la cargaison qui ne semble pas avoir subi de perturbations notables depuis le naufrage. Malgré l'absence d'une véritable fouille, il a donc été possible de tirer de nombreux renseignements de l'exceptionnel chargement du navire. Les quelques prélèvements d'amphores Oberaden 74 et d'objets appartenant à la dotation de bord ont permis de préciser la datation du naufrage, aux environs de l'époque augustéenne, et de proposer des hypothèses sur le port de départ et sur la route empruntée par le navire. Un prélèvement de la cargaison de lingots, ainsi que I'identification de différents modules, éclairent aussi de façon inédite les routes du commerce de l'étain en Méditerranée à l'époque romaine. Mais c'est avant tout l'importance quantitative de cette cargaison, estimée à environ quarante tonnes, qui est remarquable puisque cela fait de Capo Sagro 2 le plus important témoignage connu d'un chargement de métaux de la période romaine.
\end{abstract}

\section{Mots-clés}

Amphore, Corse, Époque romaine, Épave profonde, Étain, Méditerranée, Métaux, Navigation, Route commerciale
CAPO SAGRo 2: A ROMAN WRECK LOADING TIN INGOTS 500 METERS DEPTH

\section{Abstract}

The Capo Sagro 2 shipwreck lies at a depth of almost 500 metres off the cape of the same name some miles to the north-east of the coast of Corsica. Discovered by chance, a number of remotely operated dives to verify the site quickly demonstrated the unusual nature of the cargo, which is very largely composed of ingots of tin.

A photogrammetry survey of the clearly visible mound on the surface of the site revealed the relative homogeneity of the cargo, which does not appear to have suffered serious disturbance since the sinking. Despite the lack of a real excavation it has been possible to extract a lot of information about the ship's exceptional load. The lifting of a few Oberaden 74 amphorae and some objects from the crew equipment have dated the sinking to within the Augustan period and led to theories as to the port of departure and route taken by the ship. The identification of different forms of ingot and an examination of the sample that was the object of a targeted lifting have shed new light on the trade routes of tin in the Mediterranean. It is, however, above all the very size of this cargo, estimated at around forty tons, which is remarkable, since it makes the Capo Sagro 2 the largest known cargo of metal in the Roman period.

\section{Key words}

Amphora, Corsica, Roman period, Deep-water shipwreck, Tin, Mediterranean sea, Metals, Navigation, Trade route
Les études sur le commerce antique ont pu bénéficier ces dernières années des nouvelles données apportées par les recherches archéologiques sous-marines suite à l'exploration des épaves à grande profondeur. L'importance de ces sites, relevée par Patrice Pomey déjà au milieu des années 1980 (Pomey 1985, p. 61-63), est désormais une évidence pour la communauté archéologique $^{1}$. De fait, les épaves à grande profondeur pour la plupart encore bien conservées, sinon intactes, livrent en effet une quantité de données considérable, qui plus est d'une qualité remarquable.

1. Sur ces sujets, il suffit de renvoyer aux réflexions déjà développées dans Long 1998, qui retrace un tableau des recherches en eaux profondes et de leurs problématiques jusqu'à la fin des années 1990. Sur les avancées des premières années 2000 , dues surtout aux progrès des recherches dans le domaine de la documentation par photogrammétrie, grâce principalement à l'arrivée de la photographie numérique et au développement des nouveaux logiciels qui lui sont liés, voir Long et al. 2002 et Long, Drap 2010. Sur les recherches les plus récentes voir L'Hour 2014, Drap et al. 2015, Cibecchini 2015.
Le développement technologique a également rendu de plus en plus accessibles ces épaves avec des moyens logistiques et financiers qui, bien qu'importants, commencent à être à la portée des archéologues.

En Corse, les recherches dans les grands fonds ne débutèrent véritablement que dans les années 2000 avec les découvertes et les expertises conduites par la Comex sur des épaves antiques et contemporaines situées à une profondeur ne dépassant pas 100 mètres $^{2}$. Ces expertises rapides se limitèrent à la réalisation de vidéos et au prélèvement de quelques objets, le plus souvent sans la présence d'un archéologue. Il faudra attendre 2013 pour que le Drassm puisse organiser une série de campagnes de recherche et de documentation systématiques. Entre 2013 et

2. Des épaves découvertes anciennement dans les eaux de l'île, seule l'épave Sud-Perduto 1 dépasse les -60/-70 mètres qui sont atteints en plongée à l'air par certains professionnels, comme les corailleurs. Cette épave, à -90 mètres, avait été découverte par hasard en 1980 par le GISMER de la Marine Nationale qui avait réalisé quelques clichés et prélevé des amphores avec le sous-marin Griffon; voir dernièrement Cibecchini 2015 et 2016 avec bibliographie précédente. 


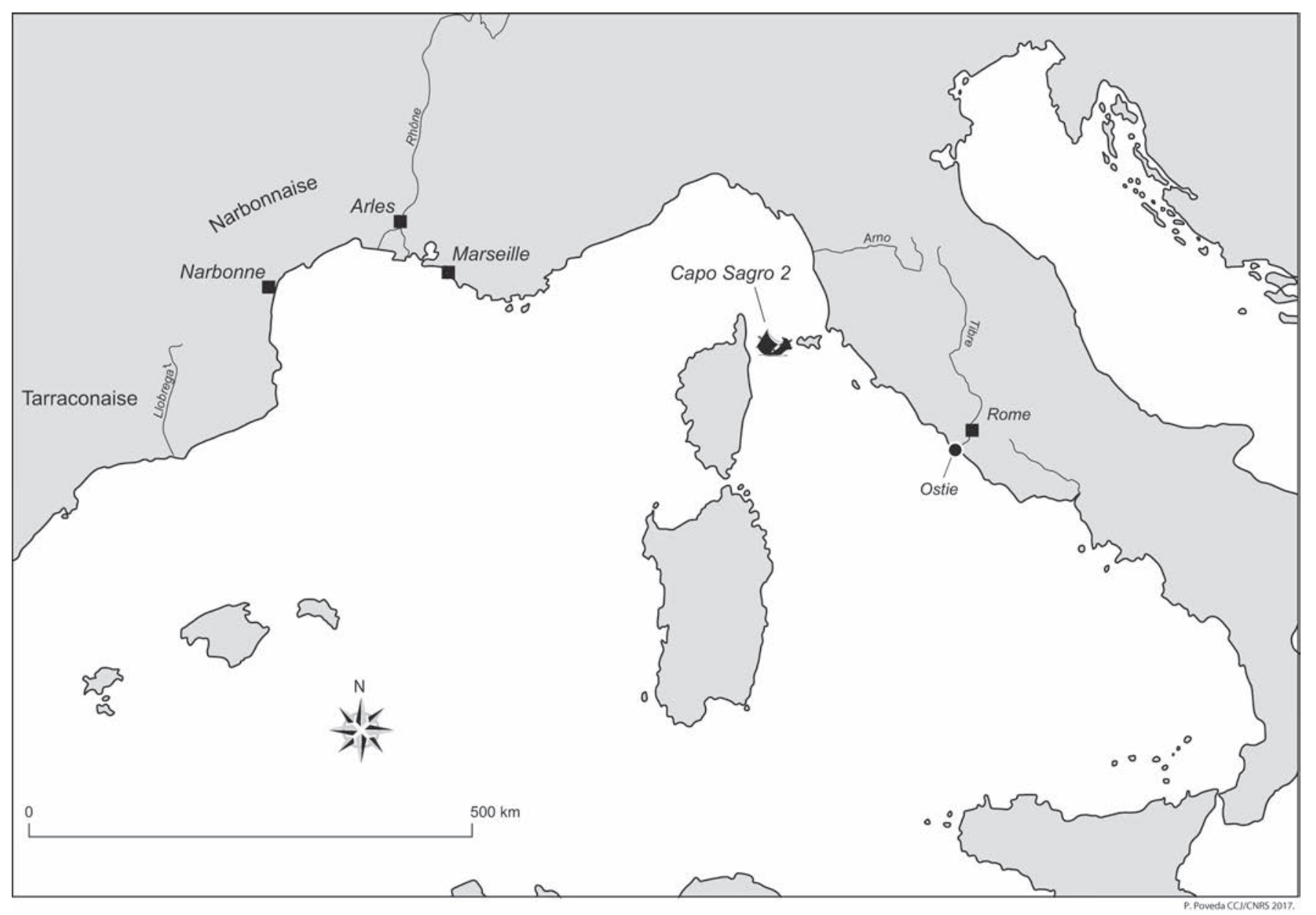

Fig. 1 : Localisation de l'épave Capo Sagro 2 (P. Poveda).

2016, en général avec l'appui du navire de recherche André Malraux, plus d'une vingtaine d'épaves localisées entre 100 et 500 mètres de profondeur ont été progressivement expertisées et documentées au large de la côte orientale de la Corse.

Ces recherches récentes ont mis encore plus en évidence la richesse de ces épaves mais aussi leur grande fragilité, surtout dans le cas des épaves antiques (L'Hour 2014, p. 158-159 ; Cibecchini 2015).

Pour ces raisons et malgré des difficultés techniques non négligeables, en 2015 nous avons décidé d'effectuer avant tout des couvertures photogrammétriques complètes de trois épaves antiques particulièrement remarquables pour leur intérêt scientifique et leur excellent état de conservation. L'épave Capo Sagro 2, chargée d'une cargaison assez exceptionnelle de lingots d'étain associés à du vin et à d'autres marchandises d'identification difficile, est la plus profonde des trois $^{3}$ (fig. 1). Cette épave a été choisie pour faire l'objet d'une étude plus approfondie en raison de son état de conservation remarquable et pour l'importance des données qu'elle peut fournir sur le commerce, encore peu connu, de l'étain. En outre, les études effectuées sur Capo Sagro 2, malgré leur

3. Les deux autres épaves sont Aleria 1, un navire marchand avec une cargaison très hétérogène en provenance de Rome/Ostie datée du règne d'Hadrien (Cibecchini 2015 et 2016) et Cap Corse 2, avec une cargaison exceptionnelle de verre brut et de vaisselle en verre datée du début du $\mathrm{II}^{\mathrm{e}}$ s. ap. J.-C. (Fontaine, Cibecchini 2014). En 2016, trois autres épaves antiques, Capo Sagro 3 et 4 et Furiani 1, ont fait l'objet de couvertures photogrammétriques. caractère préliminaire, viennent compléter celles actuellement en cours sur une autre épave plus tardive mais également chargée d'étain, Bloscon 1, découverte à Roscoff en Bretagne en 2015 et fouillée par le Drassm ${ }^{4}$.

\section{DÉCOUVERTE ET HISTORIQUE DES OPÉRATIONS SUR LE SITE}

L'épave Capo Sagro 2 a été découverte pendant une courte campagne (trois jours et demi) de prospections par grands fonds, effectuée en collaboration avec le navire Octopus de Paul Allen en juin 20145. Les prospections ont été effectuées avec un robot sous-marin autonome (AUV, Bluefin Robotics) apte à naviguer jusqu'à 2500 mètres de profondeur et qui a couvert deux zones au large du Cap Corse, l'une d'environ 4,5 km sur 1,5 km avec 13 transects espacés de manière alternée

4. Fouille dirigée par Olivia Hulot (Drassm), en 2015 et 2017. De l'épave ont été prélevées environ six tonnes d'étain, en lingots de formes différentes, en majorité discoïdes ou rectangulaires à section plano-convexe, pour une cargaison restituée d'environ 8 tonnes (Hulot 2017). Nous remercions Olivia Hulot pour ces informations.

5. Le navire Octopus est notamment lourdement équipé pour la recherche en eaux profondes. Nous avons répondu favorablement à la proposition de Paul Allen, qui voulait profiter d'un séjour prolongé en Méditerranée pour tester le nouveau AUV et pour mettre en place une collaboration avec le Drassm. 
de 400 et $350 \mathrm{~m}$, l'autre d'environ $18 \mathrm{~km}$ sur $4 \mathrm{~km}$ divisée en 19 transects de 200 x 500 m. Dans cette dernière, une petite anomalie a attiré notre attention et nous avons procédé à sa vérification avec un ROV Work Class (- 3000 mètres) armé de deux bras. Cette anomalie s'est révélée être celle d'une épave antique, un navire qui présentait un tumulus compact formé par des objets mal identifiables, de forme rectangulaire ou, moins fréquemment, ellipsoïdale et quelques amphores éparses autour du tumulus. Grâce à cette opération nous avons pu avoir une première idée des dimensions du gisement et de la cargaison en prélevant un échantillon ciblé du mobilier archéologique. Le ROV a remonté en effet une cruche avec filtre et bec verseur, un mortier/pelvis, une grande coupe en céramique commune, un bord de grande assiette en sigillée arétine et enfin un fragment de lingot. Ce fragment nous a permis de comprendre que le tumulus était composé de lingots d'étain, qui ont subi un processus de « cristallisation » de la matière en raison de leur permanence dans l'eau salée et, surtout, de la faible température de l'eau à - 500 mètres.

En mai 2015 une courte opération de documentation des épaves à grande profondeur a été menée par le Drassm au large de la côte orientale de la Corse ${ }^{6}$. L'objectif prioritaire était d'effectuer une couverture photogrammétrique sur une série d'épaves antiques. En second lieu, nous voulions prélever d'autres échantillons très ciblés de mobilier. Sur Capo Sagro 2, les deux objectifs ont été atteints, à savoir une couverture photogrammétrique de grande qualité et le prélèvement d'une amphore Oberaden 74 presque complète.

\section{LA COUVERTURE PHOTOGRAMMÉTRIQUE}

Le levé photogrammétrique a été réalisé à l'aide du ROV Work Class, Perseo GTV (Société Copetech). Un appareil reflex numérique Nikon D810 équipé d'un objectif AF Nikkor $20 \mathrm{~mm}$ a été utilisé pour l'acquisition des images. Cet appareil était situé dans un caisson étanche, fixé sur le ROV, capable de supporter des pressions jusqu'à $1500 \mathrm{~m}$ de profondeur. Ce caisson était orientable de $0^{\circ}$ à $30^{\circ}$ par rapport à la verticale. Les couvertures ont été faites avec un angle de $0^{\circ}$. L'élément le plus important et décisif pour la réalisation d'une couverture de qualité à cette profondeur a été la lumière : nous avons expérimenté des éclairages spéciaux, montés sur le ROV sur de longs bras latéraux, avec une puissance de 250000 Lumen chacun.

La couverture photogrammétrique de Capo Sagro 2 a été la seconde réalisée durant cette mission et se trouve sans aucun doute être la plus aboutie et donc la meilleure des trois. Les excellentes conditions de visibilité du site, malgré la grande profondeur (-500 m), et l'absence de problèmes techniques y ont largement contribué.

Le protocole de prise de vue était similaire au protocole standard en photogrammétrie. L'acquisition devait s'effectuer par bandes parallèles orientées selon la plus grande longueur de l'épave, avec un recouvrement interbande de $80 \%$ et un recouvrement intrabande de $20 \%$ des photos. Deux bandes complémentaires ont été réalisées aux extrémités du site et

6. L'opération, sous la direction de F. Cibecchini, avec l'appui du navire de recherche André Malraux, s'est déroulée du 14 au 18 avril 2015 et a vu la collaboration en particulier de Vincent Creuze (LIRMM, UMR 5506), Denis Degez (Drassm), Frédéric Osada, Maxime Seguin (Inrap). perpendiculairement aux autres afin de contraindre le calcul d'aérotriangulation. La hauteur de vol se trouvait entre quatre et deux mètres au-dessus du fond.

Le respect de ces contraintes s'est avéré particulièrement difficile à réaliser, principalement en raison de la faible vitesse d'avancement du ROV. La restitution a été effectuée avec le logiciel PhotoScan par Maxime Seguin (Inrap). L'orthophotographie a été réalisée avec 654 clichés sur les plus de 1000 pris sur le site (fig. 2). La reconstitution 3D par nuages de points de l'épave a permis de réaliser des coupes transversales et longitudinales du site.

La qualité remarquable de l'orthophotographie nous permet de mesurer le gisement et même un grand nombre d'objets, avec une marge d'erreur très réduite de l'ordre du centimètre. La grande qualité des images, grâce surtout au système d'éclairage, a permis d'observer plusieurs détails et de procéder à l'identification de la plupart des objets visibles en surface.

\section{LE GISEMENT : CARACTÉRISTIQUES, DIMENSIONS ET COMPOSITION DE L'ÉPAVE ET DE LA CARGAISON}

Pratiquement intacte, l'épave est orientée S/SE-N/NO et occupe une surface d'environ 16,5 mètres de longueur sur 9 mètres de largeur dans son extension maximale. Elle se compose d'un tumulus central assez compact, composé essentiellement de lingots d'étain. La zone occupée par le tumulus et le groupe le plus dense d'amphores qui l'entourent dépasse à peine les neuf mètres de longueur. À environ quatre ou cinq mètres vers le sud-est, selon l'axe de l'épave, sont visibles trois amphores avec un lot de céramiques de table et quatre jas d'ancre en plomb, groupés deux par deux et répartis de part et d'autre de l'axe longitudinal de l'épave. Cette zone devait correspondre à l'avant du bateau, mais l'espace entre celle-ci et le tumulus de lingots et le groupe d'amphores semble complètement vide. Cette configuration n'est pas facilement explicable. Une hypothèse possible est que cette partie de la cale était occupée par des marchandises périssables, comme des sacs de blé par exemple, qui n'ont pas laissé de traces visibles, du moins en surface ${ }^{7}$. La présence à l'extrémité nord-ouest d'un tuyau, vraisemblablement en plomb, qui semble sortir d'un côté et de l'autre du tumulus ${ }^{8}$, renforce notre hypothèse d'orientation de l'épave. En outre, ce tuyau se trouve en correspondance avec un espace rectangulaire d'environ un mètre sur $0,85 \mathrm{~m}$, bien délimité par les lingots sur trois côtés, au centre du tumulus et parfaitement placé sur l'axe longitudinal de l'épave. Il s'agit très probablement de l'espace réservé à la pompe de cale qui était habituellement localisée entre le quart et le tiers arrière du navire.

Le tumulus central se compose essentiellement de grands lingots, en apparence rectangulaires ou allongés aux extrémités arrondies, tous vraisemblablement d'étain, comme le fragment récupéré en 2014 (fig. 3). L'analyse par fluorescence X portable

7. Il serait nécessaire d'effectuer un sondage limité avec prélèvement d'une carotte de sédiment au centre de cette zone apparemment vide pour essayer de comprendre si le navire transportait effectivement un autre type de cargaison et de quelle marchandise il pourrait s'agir.

8. Il dépasse d'environ un mètre du tumulus sur le côté ouest pour $1,70 \mathrm{~m}$ de tuyau visible sur le côté est. 


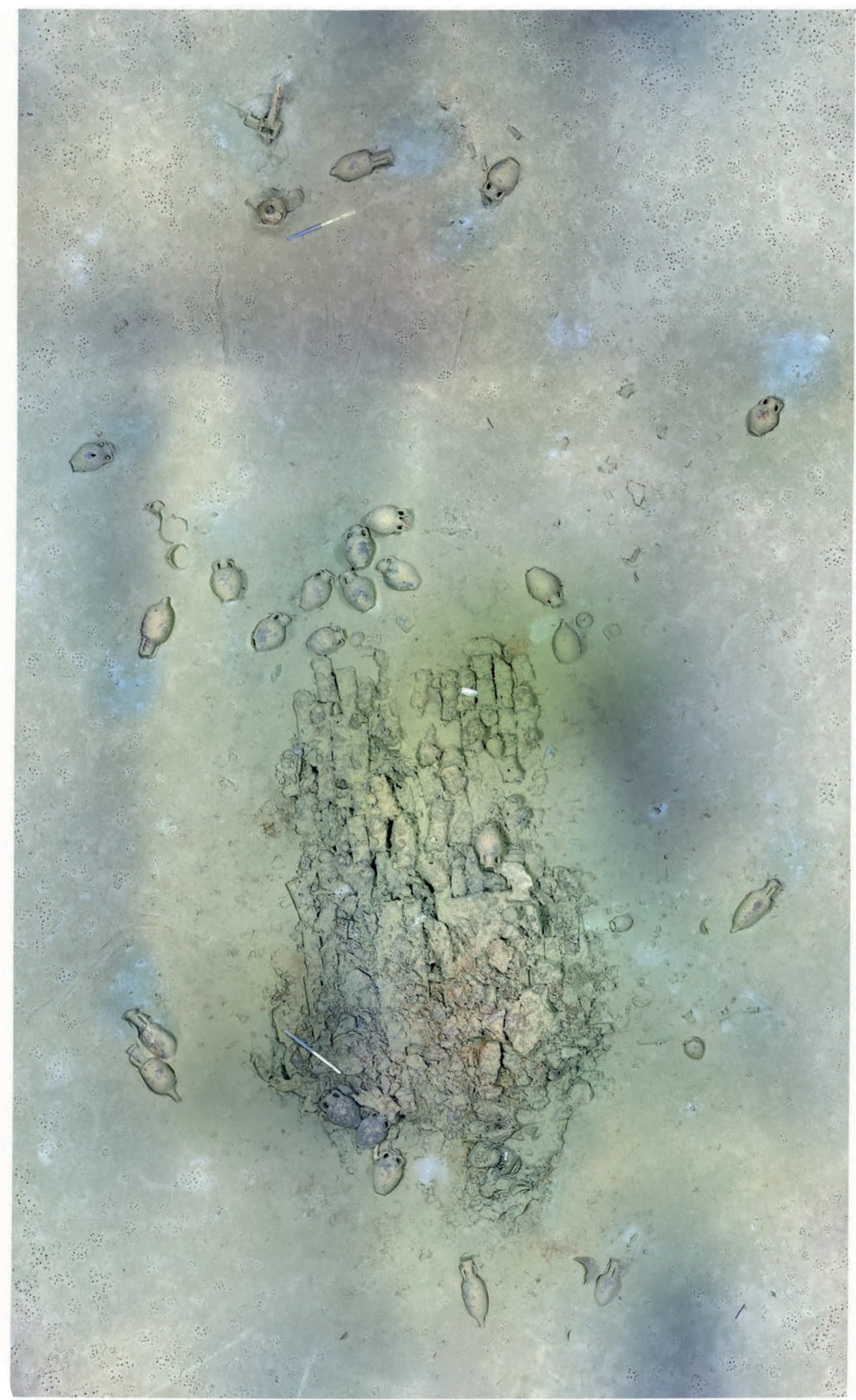

Fig. 2 : Orthophotographie de l'épave Capo Sagro 2 en 2015 (clichés Drassm, élaboration M. Seguin, Inrap). 


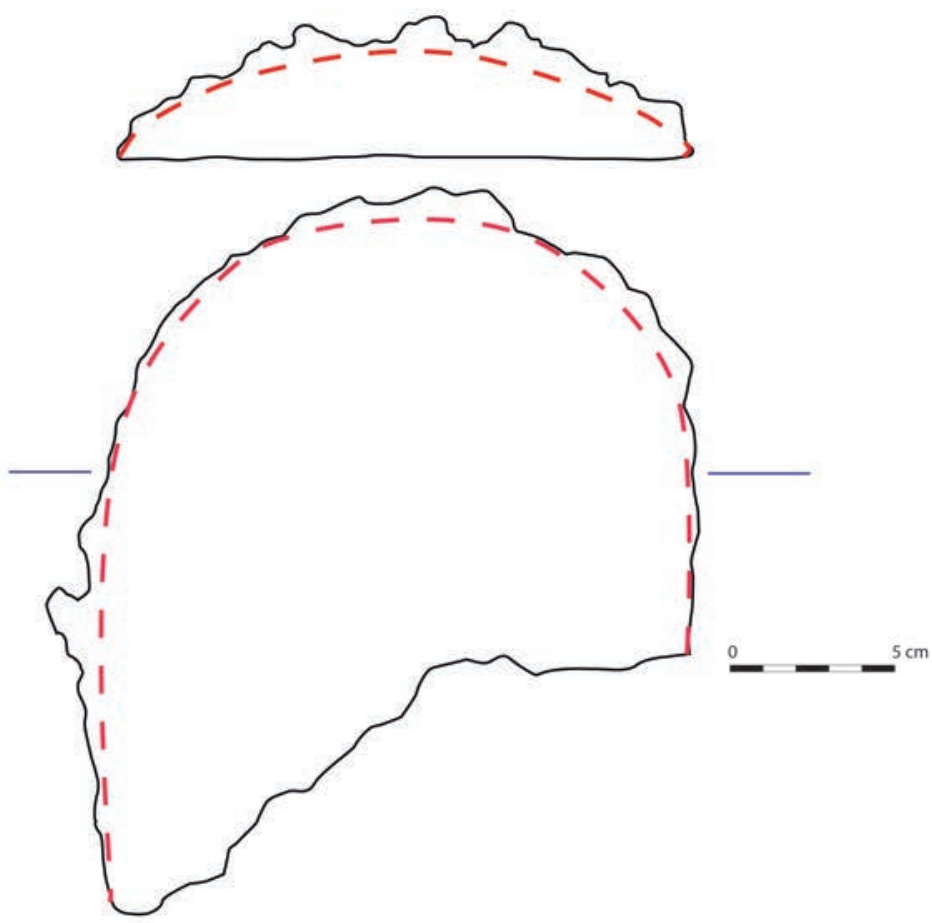

Fig. 3 : Fragment du lingot récupéré en 2014 (cliché et DAO F. Cibecchini).

effectuée sur cet échantillon par Cécile Le Carlier (Archéoscience - CNRS, Rennes) a confirmé qu'il s'agit d'étain pur à $98 \%{ }^{9}$. Au centre du tumulus on remarque une espèce de bande vide longitudinale, d'une vingtaine de centimètres de largeur, qui partage en deux l'amas des lingots et semble suivre précisément l'axe longitudinal du navire. Il s'agit vraisemblablement d'un creux révélant à cet endroit la présence, sous le chargement, d'un élément structurel d'importance pour le navire, tel qu'une carlingue.

Les dimensions du tumulus de lingots sont de 6,40 m maximum de longueur sur 4,10 m à son point le plus large (extrémité nordouest) et de 3,30 m à son point le plus étroit (extrémité sud-est). L'orthophotographie nous a permis de dénombrer un peu plus de 80 lingots dans les couches visibles du tumulus.

Trois types de lingots sont reconnaissables dans ce tumulus :

1 - des lingots allongés, rectangulaires, dont l'orthophotographie de la couche supérieure nous montre essentiellement la face inférieure. Il est difficile de déterminer avec assurance leur profil, qui semble toutefois plutôt écrasé que bombé. L'épaisseur de ces lingots a pu être mesurée sur plusieurs exemplaires du côté ouest et du côté est ; elle est comprise entre 3 et $6 \mathrm{~cm}$. Les dimensions, dans les couches bien visibles, sont assez régulières : $61 \mathrm{~cm} \times 23 \mathrm{~cm}$; certains lingots atteignent toutefois une longueur comprise ente 68 et $71 \mathrm{~cm}$.

2 - des lingots allongés, plus ou moins ovoïdes, plats, aux extrémités arrondies et à la face supérieure également arrondie, comme le lingot qui a été échantillonné (fig. 3). Ils sont plus petits que les autres : les dimensions des exemplaires que nous avons pu mesurer sont $40 / 50 \mathrm{~cm}$ par $22 / 18 \mathrm{~cm}$ avec une épaisseur de $3 / 4 \mathrm{~cm}$ maximum.

9. Plus précisément : (Sn) 98,62 étain, (Ti) 0,544 titane, (Fe) 0,646 fer, (AS) 0,024, arsenic (Cd) 0,026 cadmium, (In) 0,114 indium.

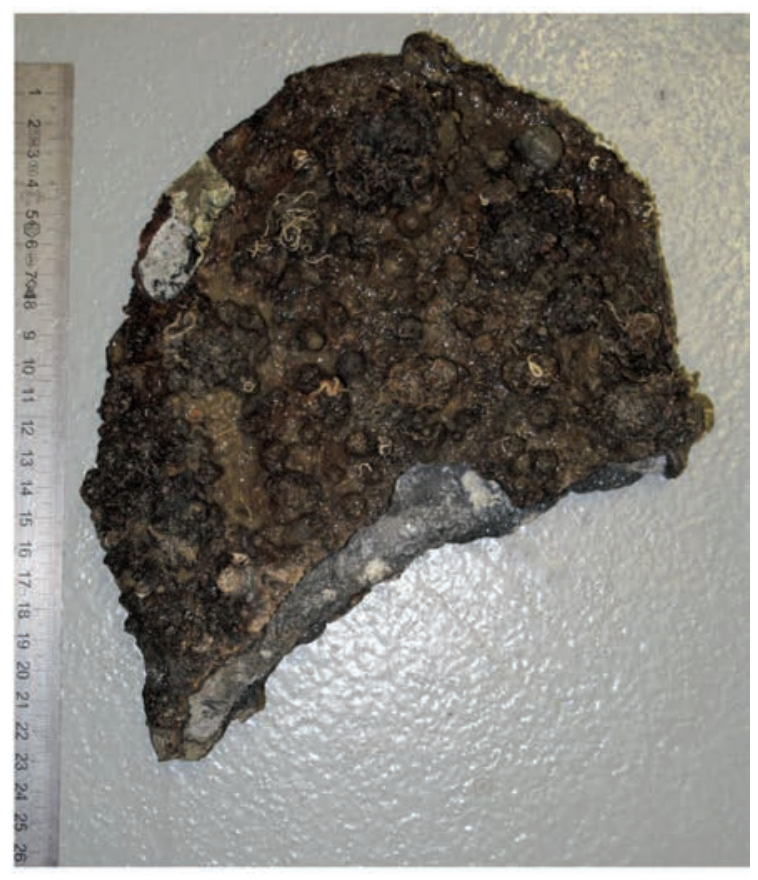

3 - des lingots plus trapus, rectangulaires mais courts, et apparemment présentant un profil tronco-pyramidal et une épaisseur bien plus importante. Leurs dimensions sont de $32 / 36 \mathrm{~cm}$ de longueur pour une largeur de $22 / 23 \mathrm{~cm}$; l'épaisseur est comprise entre 11 et $14 \mathrm{~cm}$.

Globalement, donc, ils se rapprochent des lingots des épaves Bagaud 2 et Mèdes 1, toutes deux datées de la fin de la République. On n'observe pas de dispositifs pour faciliter leur manutention, comme des anses ou autres appendices de préhension, comme sur les lingots des épaves Cala Rossano, Lavezzi 2, Plage de Losari et Port-Vendres 2, dont ils sont du reste complètement différents.

Le tumulus peut être divisé selon l'axe de la structure longitudinale disparue en deux moitiés : la partie est, d'une largeur max. d'environ 1,85 mètre, semble être plus abîmée et est moins lisible que la partie ouest, d'une largeur max. d'environ 1,65 mètre ${ }^{10}$. En outre, une autre partition possible du tumulus est visible transversalement et correspond à environ sa moitié nord-ouest ${ }^{11}$, immédiatement au nord de la seule amphore Oberaden 74 qui gît sur les lingots. Le tumulus est ainsi partagé en quatre secteurs : NO ; NE; SO et SE. Le nuage de points 3D montre clairement, et notamment dans la partie ouest, que cette zone comptait au moins une couche, sinon deux, de lingots de plus que dans la moitié sud. En effet, nous pouvons reconnaître, hors sédiments, au moins quatre niveaux de lingots dans les secteurs NO et NE et au moins trois dans le SO. Dans les secteurs NO/NE les lingots de la couche supérieure sont en grande

10. La présence d'au moins deux filets de pêche et la fragmentation des trois amphores Oberaden 74 à son extrémité nord semblent indiquer que le désordre visible dans cette zone, surtout au nord, est dû au passage d'un chalut, mais nous n'avons aucune autre trace claire d'un possible chalutage de cette épave.

11. Dimensions moitié nord : env. $3 \mathrm{~m} \times 3,60 \mathrm{~m}$ max. ; moitié sud : env. $3,40 \mathrm{~m} \times 3,50 \mathrm{~m} \max$. 
majorité du type 1, apparemment soudés entre eux à plusieurs endroits. Si dans le secteur NE nous pouvons reconnaître assez facilement sept lignes ou rangées de lingots, plus ou moins soudés entre eux, seulement trois sont bien reconnaissables sur le côté NO, tandis que l'extrémité ouest de ce secteur paraît plus chaotique. Les lingots de la couche superficielle sont ici en mauvais état de conservation et ils semblent avoir été bouleversés. La lecture est encore plus complexe dans le secteur SE : si trois niveaux de lingots sont clairement identifiables à l'extrémité nord du secteur, en correspondance de la seule amphore Oberaden gisant sur le tumulus, nous ne pouvons identifier qu'une seule couche de lingots émergeant du sédiment plus au sud. Cette dernière semble toutefois composée pour la plupart de lingots de type 3, bien plus épais que les autres.

Les lingots de la couche supérieure semblent soigneusement rangés en lignes parallèles selon l'axe longitudinal du navire et ils ne paraissent pas avoir beaucoup bougé lors du naufrage, ce qui laisse penser que le navire a coulé à pic en maintenant une trajectoire directe avant de toucher le fond. Dans la zone centrale du tumulus on voit assez clairement que les lingots sont disposés en assises croisées, ce qui expliquerait pourquoi un certain nombre d'entre eux montrent des tranches très nettes. Ces lingots n'ont pas été rangés sur leur face principale, mais à l'envers, donnant ainsi à voir leur face inférieure. Une telle disposition, qui assure une plus grande stabilité au chargement, est bien attestée pour des lingots de plomb sur l'épave SudPerduto 2 (Bernard, Domergue 1991, p. 42-43).

Enfin, on observe que l'amas métallique fragmentaire visible en surface dans la partie nord du tumulus autour de l'axe longitudinal, spécialement dans le secteur NE, et dans l'espace attribué à la pompe de sentine, présente une coloration rougeâtre proche de celle produite par la rouille. L'hypothèse d'une petite cargaison de barres de fer, comme on en connaît sur les épaves Bagaud 2, Mèdes 1 et Capo Bellavista (infra), nous paraît très vraisemblable.

Le tumulus est entouré par une trentaine d'amphores éparpillées ou rassemblées en petits groupes de quelques individus. Les amphores Oberaden 74, parmi les plus nombreuses (22 NMI au total), peuvent être interprétées dans leur grande majorité comme appartenant à la cargaison du navire. Seuls les deux exemplaires visibles à l'avant de l'épave, dans la zone interprétée comme appartenant à une possible cabine ou à une zone de vie, pourraient avoir fait partie de la dotation de bord. Le groupe le plus important de ces Oberaden 74 (quatorze exemplaires) se trouve au centre de l'épave, principalement à la lisière entre le tumulus des lingots et l'espace vide qui le sépare de l'avant du navire. On pourrait ici restituer une disposition en deux rangées parallèles de sept amphores en correspondance de l'extrémité NO du tumulus des lingots. Enfin, les six dernières amphores de ce type se trouvent placées symétriquement sur bâbord et tribord à l'arrière du gisement, les trois individus situés côté ouest se révélant ainsi parfaitement conservés. Ces six amphores semblent avoir occupé des espaces bien précis.

Plus délicate est l'interprétation des quatre amphores de type Tarraconaise 1 et, plus encore, des quatre amphores Lamboglia 2. Trois amphores Tarraconaise 1 sont ainsi bien visibles, deux à l'extrémité nord, nord-est du site et la troisième à près de 2 mètres à l'est au centre du tumulus des lingots. L'attribution à ce même type d'une quatrième amphore, partiellement enfouie dans le sédiment immédiatement à l'est de l'extrémité sud du tumulus, paraît aussi envisageable.
Les amphores adriatiques sont disposées de manière presque symétrique : les deux exemplaires qui semblent les plus récents, proches du type Dressel $6 \mathrm{~A}$, se trouvent à moins de $1,5 \mathrm{~m}$ à l'ouest de la partie finale du tumulus, tandis que celles proches du type Lamboglia 2 sont également sur le côté ouest, mais au centre de l'épave pour la première et à l'avant du site pour la seconde, entre les jas d'ancre et le pelvis remonté à la surface.

L'interprétation de la fonction des trois grandes ollae avec anses en céramique commune, qui se trouvent sur le côté est du tumulus et les quelques céramiques éparpillées entre les amphores à la lisière entre les tumulus et l'espace vide, se révèle tout aussi complexe. On peut y reconnaître deux bouteilles ou lagynoi et une olpé. Un troisième lagynos est visible sous une des trois Oberaden 74 de l'extrémité nord-ouest (pour une étude détaillée, voir infra).

\section{LE MOBILIER CÉRAMIQUE}

Comme décrit ci-dessus, quelques dizaines d'amphores et quelques pièces de vaisselle en céramique accompagnaient les lingots. Certaines pièces ont été récupérées et ont été étudiées et documentées à terre. Plusieurs autres objets, bien visibles en surface du gisement, ont pu être identifiés avec une bonne marge de certitude. Au total, nous avons repéré une dizaine de pièces de vaisselle, la plupart appartenant vraisemblablement à la dotation de bord et une trentaine d'amphores. Dans la plupart des cas, nous avons pu mesurer ce mobilier dans le nuage de points 3D. Même si une marge d'imprécision dans ces mesures est sans doute à envisager, elle nous semble très faible et les dimensions calculées sont conformes à celles connues pour la majorité des productions présentes.

\section{LES AMPHORES}

\section{Les amphores Oberaden 74}

Nous avons recensé 22 exemplaires d'amphores Oberaden 74 , dont 16 entières. Un de ces exemplaires, dont le fond est manquant, a été récupéré en 2015 (fig. 5). Toutes les amphores, en majorité bien visibles en surface, sont assez standardisées et assez similaires entre elles.

Ces amphores constituent sans aucun doute une cargaison complémentaire à celle des lingots d'étain. La localisation actuelle de la majorité des exemplaires permet de restituer une disposition assez précise de ces amphores dans le navire, comme nous l'avons vu ci-dessus, en deux rangées parallèles de sept amphores pratiquement au centre du navire et deux rangées de trois amphores, symétriques, sur bâbord et tribord à l'arrière du gisement. Pour les deux exemplaires à l'avant du navire il est possible qu'ils appartiennent à la dotation de bord du navire. Malgré leur nombre réduit, nous avons ici la plus importante cargaison d'Oberaden 74 connue sur une épave dans toute la Méditerranée.

L'amphore Oberaden 74 est la première amphore à fond plat produite dans la péninsule Ibérique et, selon certains chercheurs, elle serait même à l'origine des amphores gauloises impériales (Carreras Monfort, González Cesteros 2012, p. 214 ; Rizzo 2014, p. 198). Ces amphores sont produites dans une quinzaine d'ateliers de la côte nord-est de la Tarraconaise, 


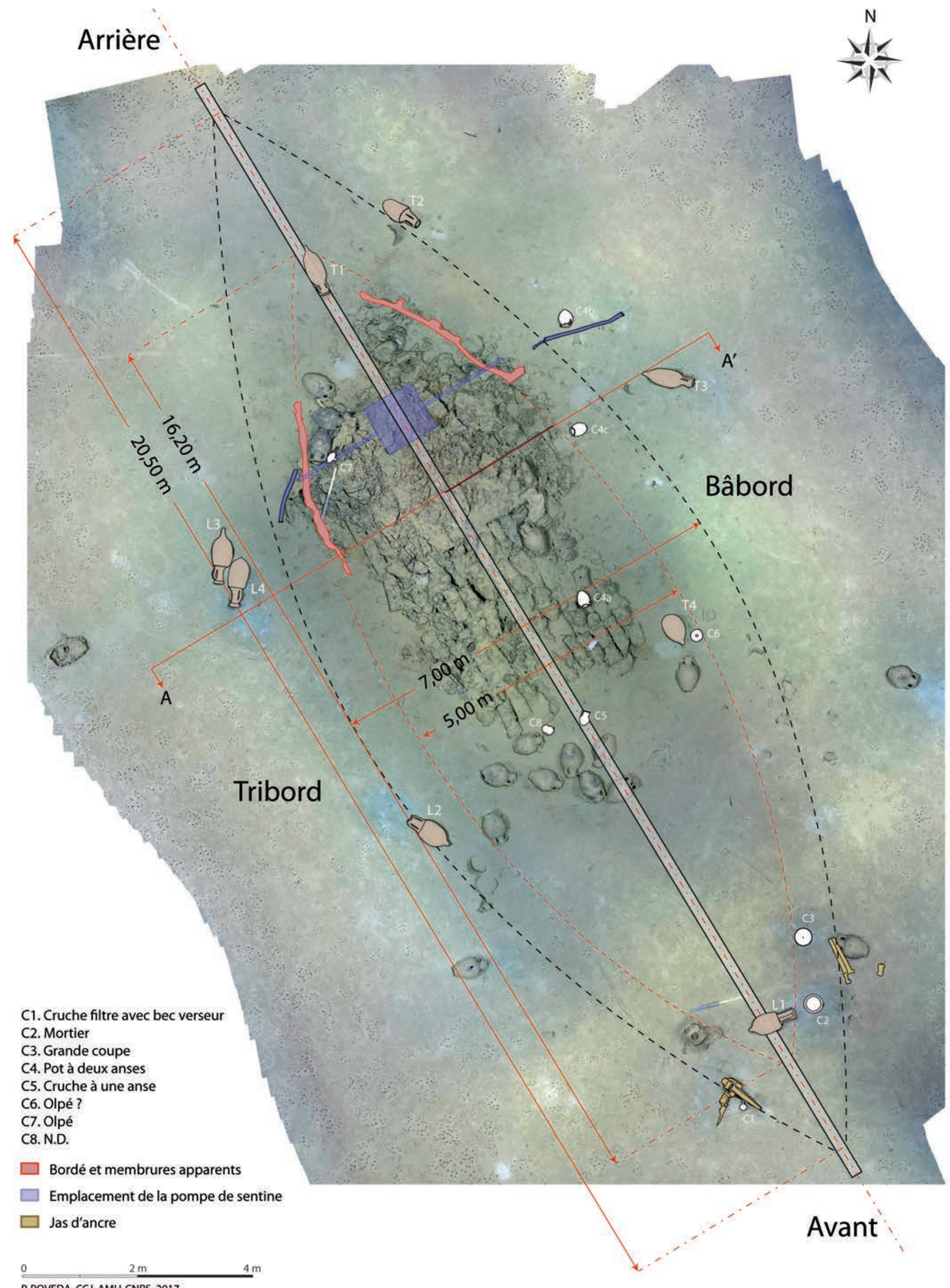

P. POVEDA, CCJ, AMU-CNRS, 2017

Fig. 4: Évaluation sur l'orthophotographie des dimensions d'origine du navire et localisation des éléments architecturaux et céramiques significatifs (élaboration P. Poveda). 


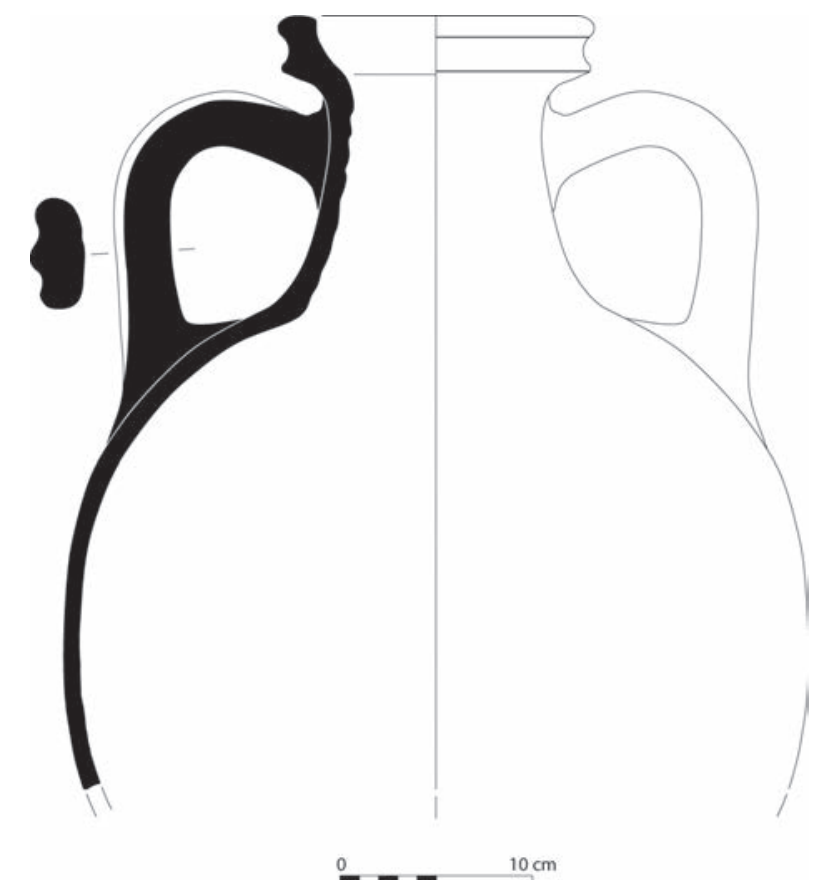

Fig. 5 : Amphore Oberaden 74, inv. 29116 (prélèvement 2016, dessin M.N. Baudrand).

même s'ils sont surtout concentrés autour du Baix Ebre, aux alentours de Tarragone et au nord de Barcelone ${ }^{12}$. Le contenu pressenti est le vin, la zone de production ayant une forte tradition viticole de bonne réputation. La forme constitue un autre indice de contenu, la plupart des amphores à fond plat transportant du vin. Il s'agit d'une production très limitée, tant du point de vue quantitatif que dans la durée. Elle est attestée entre 30 av. et 60 ap. J.-C., mais la période de plus grande diffusion de ces amphores correspond à l'époque augustéenne, plus précisément entre 20 av. et 20 ap. J.-C. ${ }^{13}$. Il s'agirait d'amphores destinées essentiellement à une diffusion fluviale, le long des cours d'eau navigables de la Gaule et, surtout, de la Germania, avec une distribution très particulière, centrée surtout sur les zones militaires du limes rhénan qui semblent constituer le marché privilégié des Oberaden 74 (Carreras Monfort, González Cesteros 2012, p. 221-223 et fig. 11 ; 2013 ; González Cesteros 2015, p. 211). Toutefois, elles semblent curieusement absentes à Fos et à Arles (Laubenheimer 2015) alors qu'elles sont présentes à Narbonne ${ }^{14}$. Elles circulent toutefois aussi en Méditerranée occidentale, bien qu'en quantités très réduites, comme en témoigne leur présence dans la région d'Alicante et à Minorque ou à Carthage (Carreras Monfort, Gonzáles Cesteros 2012, p. 223). Ces amphores sont également bien attestées à Rome, bien que toujours en quantités très réduites et résiduelles dans des contextes flaviens et antonins (Rizzo 2014, p. 198, tab. 27). Il n'est pas exclu que ces amphores transportaient du vin de qualité, provenant notamment de l'ager de Tarraco (González Cesteros 2015, en particulier p. 214).

12. Carreras Monfort, González Cesteros 2012, avec carte des ateliers en fig. 1. Cet article réunit la plupart des données disponibles sur ce type d'amphore avec une bibliographie exhaustive des études précédentes.

13. Ibid., p. 217-218.

14. Plusieurs exemplaires inédits sont attestés à Port-la-Nautique/Narbonne, information personnelle de Corinne Sanchez que nous remercions pour nous avoir fourni ces données.
Les dimensions mesurées sur les nuages de points des amphores in situ dans l'épave et celles de l'exemplaire prélevé correspondent et permettent d'envisager des amphores avec une hauteur moyenne de $63-65 \mathrm{~cm}$, un diamètre maximal de $38-39 \mathrm{~cm}$, un diamètre du bord int./ext. de 12,5/16,5 cm et un diamètre du fond de $15-18 \mathrm{~cm}$ max. D'un point de vue morphologique cette amphore trouve ses meilleures comparaisons avec l'exemplaire du campement d'Oberaden qui illustre le type 74 de Loeschke et, plus généralement, avec des cols rattachés aux productions de la vallée de l'Èbre ou découverts dans d'autres campements du limes, comme Dangstetten (González Cesteros, Carreras Monfort 2016, fig. 1 et fig. 2 ; Ehmig 2010, pl. 8 ; Carreras Monfort, González Cesteros 2012, p. 216-218, fig. 9, n. 9-11). La pâte céramique du seul exemplaire prélevé est de couleur beige à peine rosée, avec des stries couleur noisette, légèrement granuleuse, et comporte de nombreuses inclusions blanchâtres de petites dimensions. L'analyse pétrographique en lame mince effectuée par Claudio Capelli a mis en évidence la présence d'inclusions de quartz métamorphique fin et des silicates métamorphiques. Malgré sa couleur claire, cette pâte pourrait correspondre davantage aux productions de l'Èbre qu'à celles de Tarragone. En revanche, la pâte n'est pas compatible avec une fabrication dans la région de Barcelone ${ }^{15}$. L'épave Capo Sagro 2 rouvre donc la discussion autour de l'hypothèse d'une possible distribution de ces amphores en Méditerranée occidentale par la même route que celle empruntée par les amphores de Bétique et de Tarraconaise, contemporaines ou légèrement plus tardives, par les bouches de Bonifacio et en direction de Rome (Carreras Monfort, González Cesteros 2012, p. 223). Une hypothèse rendue fragile également par l'absence de ce type d'amphore dans les nombreuses épaves et zones de mouillage connues dans la zone du détroit.

\section{Les amphores Tarraconaise 1}

Quatre amphores éparpillées le long du côté est et à l'arrière du navire ont été identifiées comme des Tarraconaise 1 (fig. 4, T.1 - T.4, fig. 6, T.1 - T.3 $3^{16}$ ). Cette identification est moins assurée pour l'exemplaire T.4 (fig. 4, T.4), presque au centre du navire, dont seul le corps et le fond sont visibles.

Les amphores Tarraconnaise 1, appelées pendant longtemps aussi léétaniennes, sont produites le long de la côte de l'ager Tarraconensis, autour de Calafell et jusqu'à l'actuelle Lloret de Mar (Gérone). Cette production débute dans la seconde moitié du I ${ }^{\mathrm{er}} \mathrm{s}$. av. J. -C., est sans doute déjà bien attestée à partir des années 40 av. J.-C. et perdure jusqu'au tout début du $\mathrm{I}^{\mathrm{er}} \mathrm{s}$. ap. J.-C., même si elle semble en forte décadence dès les années 20 av. J.-C. Le floruit se situe entre 40 et 20 av. J.-C. (López Mullor, Martín Menéndez 2008b, p. 697). Ces amphores sont considérées comme des conteneurs vinaires. Elles sont surtout diffusées en Gaule, encore plus qu'en Catalogne, principalement le long du Rhône et à travers l'axe Aude-Garonne, à partir de Portla-Nautique/Narbonne, même si elles restent minoritaires par rapport aux Pascual $1^{17}$.

15. Nous remercions Horacio González Cesteros qui a confirmé, par une analyse autoptique, la probable provenance de la vallée de l'Èbre.

16. Sur ce type, voir la mise au point de López Mullor, Martín Menéndez 2008b, p. 693-698, avec bibliographie précédente, López Mullor, Martín Menéndez 2008a, p. 44-54 et Mirò 2016.

17. Ibid., p. 697 ; Sanchez 2015, en part. p. 168 ; Laubenheimer 2015 ; Nieto, Raurich 1998, p. 114 

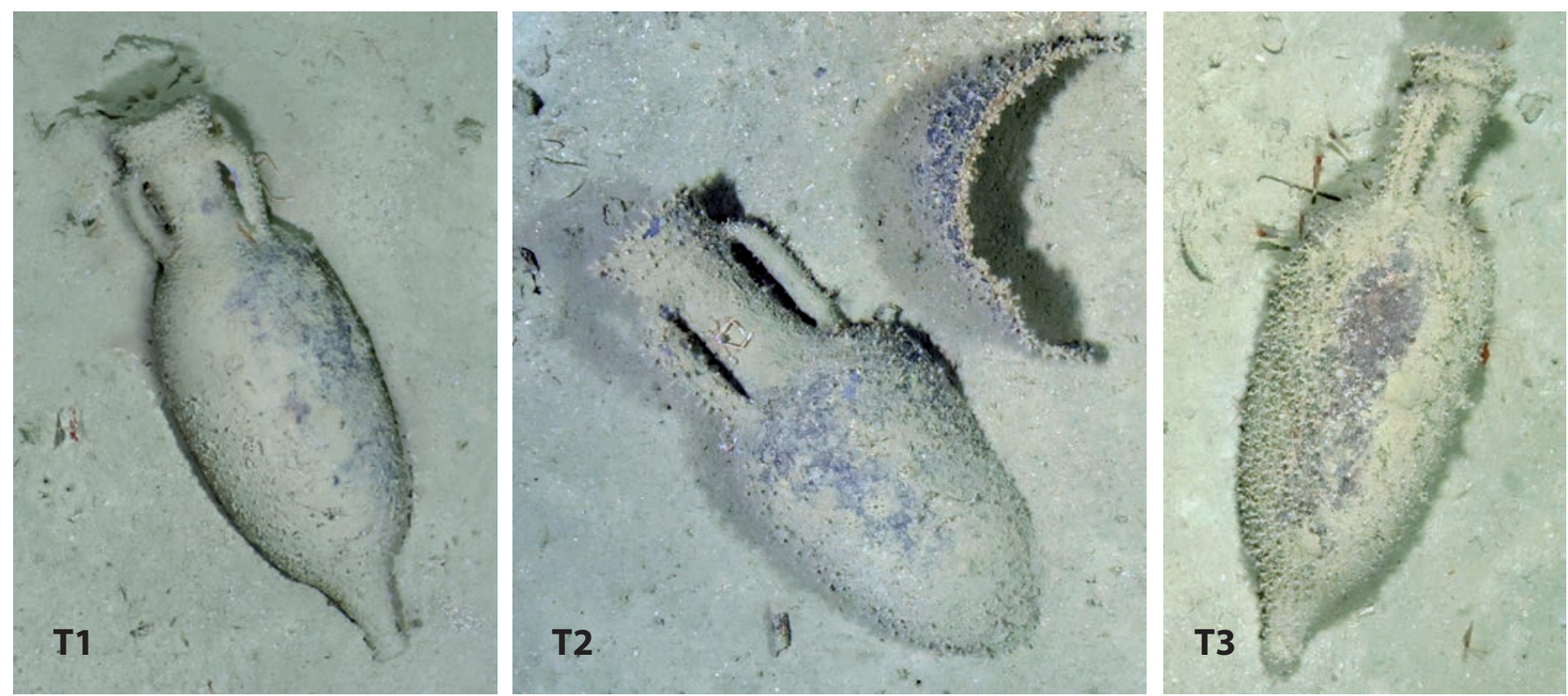

Fig. 6 : Amphores Tarraconaise 1 (T1-T3) in situ (clichés Drassm).
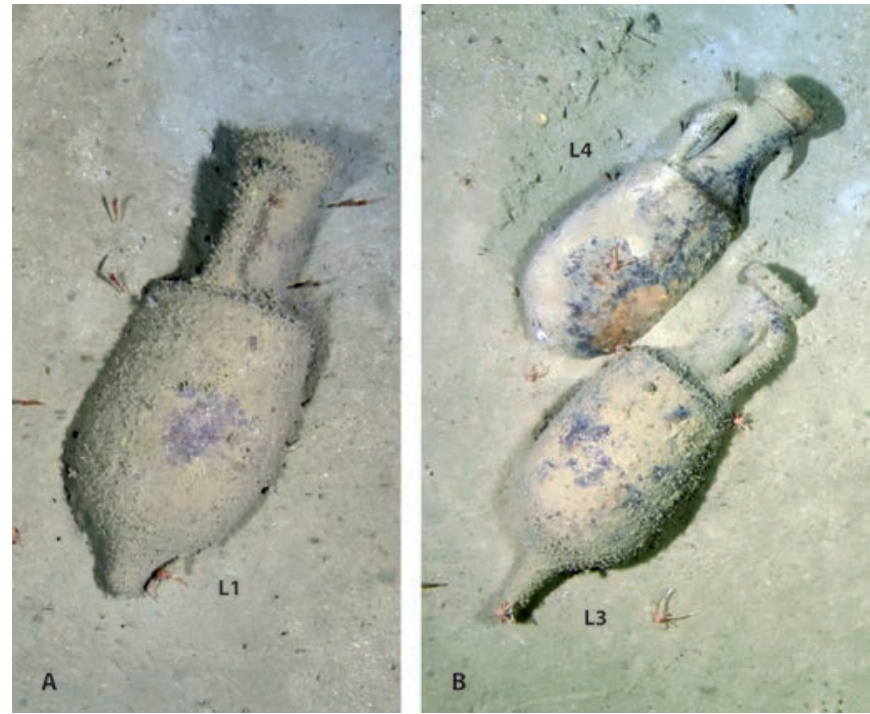

Fig. 7 : Amphores Lamboglia 2 (L1, L3, L4) in situ (clichés Drassm).

D'un point de vue morphologique, cinq variantes (Tarraconaise 1A-1E) ont été différenciées par les chercheurs (López Mullor, Martín Menéndez 2008a, fig. 5 et 6 ; id. 2008b, fig. 2). Les amphores T.1 et T.2, les mieux visibles et facilement mesurables, semblent comparables respectivement aux Tarraconaise $1 \mathrm{E}$ et $1 \mathrm{~A}^{18}$. L'amphore T.3, partiellement visible, semble correspondre à une Tarraconaise $1 \mathrm{~A}$, plus ovoïde et avec une pointe plus réduite. L'association des deux variantes se retrouve sur l'épave de Cala Bona (Cadaqués), datée entre 50 et 30 av. J.-C. (López Mullor, Martín Menéndez 2008b, p. 697).

18. Les amphores T.1 et T. 3 ont des mesures pratiquement identiques, $\mathrm{H}$. $89 / 90 \mathrm{~cm}$, diam. max de 30/31 cm, H. lèvre 4,3 cm env., mais le corps de T.1 semble légèrement plus fuselé, la lèvre est en bandeau lisse et la pointe plus tronconique tandis que la T. 3 montre une lèvre moulurée, typique des Tarraconaise 1A. Il est encore plus difficile d'essayer de trancher entre 1A et 1E pour l'exemplaire T.2 dont seule la partie supérieure est visible.
Une comparaison pourrait être également établie entre nos amphores et l'une des Tarraconnaise 1 de l'épave Sud Caveaux 1 (Long 1998, fig. 4, e) même si cette dernière est plus haute.

\section{Les amphores Lamboglia 2/Dressel 6A}

Quatre exemplaires peuvent être attribués à ce type. Deux d'entre eux se rapprochent des Lamboglia 2 classiques du ${ }^{\text {er }} \mathrm{s}$. av. J.-C., tandis que les deux autres sont attribuables à une variante peut-être plus tardive, une forme de transition entre les Lamb. 2 et les Dressel 6A. La Lamboglia 2 « classique » trouve place à l'avant du navire' ${ }^{19}$, l'amphore sans lèvre localisée à quelques mètres de distance vers le nord-ouest est d'identification plus incertaine ${ }^{20}$ (fig. 4, L.1 et L.2, fig. 7, A). Les deux autres amphores sont côte à côte quelques mètres plus au nord-ouest, à la hauteur de la pompe de cale (fig. 4, L.3 et L.4, fig. 7, B). La lèvre plus massive et carrée (L.3) ou en bandeau évasé (L.4), la pointe assez longue, bien visible dans une des deux amphores (L.3), l'épaule avec une carène très marquée ne laissent guère de doutes sur leur attribution à des Lamboglia 2/Dressel 6A ${ }^{21}$. Les Lamboglia 2 sont des amphores à vin produites entre la fin du $\mathrm{II}^{\mathrm{e}}$ et la fin du $\mathrm{I}^{\mathrm{er}} \mathrm{S}$. av. J.-C. dans les nombreux ateliers de la côte italienne de l'Adriatique centrale (Picenum et Aemilia). Une forme proche des gréco-italiques, qui a fort peu circulé, est attestée parmi les productions de Brindes ; les productions de la côte tyrrhénienne sont anecdotiques ${ }^{22}$. Les Dressel 6A représentent l'évolution morphologique des Lamboglia 2, qu'elles

19. Dimensions mesurées : L.1 : H. 85 cm, diam. panse env. $33-35$ cm. La lèvre, bien visible, est triangulaire, peu haute et la pointe est assez courte.

20. Le profil du corps et des anses et les dimensions de cette amphore semblent toutefois laisser peu de doutes sur son attribution à une Lamboglia 2 très proche de la précédente. Dimensions : L.2 : H. $85 \mathrm{~cm}$; diam. panse $38 \mathrm{~cm}$

21. Dimensions mesurées : L. 3 : H. $96-97 \mathrm{~cm}$, diam. panse env. $35 \mathrm{~cm}, \mathrm{~h}$. pointe $17 \mathrm{~cm}$, H. lèvre $4,5 \mathrm{~cm}$; L. 4 : H. max. visible $85,5 \mathrm{~cm}$, diam. panse env. $35 \mathrm{~cm}, \mathrm{H}$. lèvre $6,5 \mathrm{~cm}$.

22. Sur la chronologie et les lieux de production des amphores Lamboglia 2 et Dressel 6A, voir la mise à point de Rizzo 2014, p. 120-123, avec bibliographie. Pour la typologie des lèvres, Forti, Paci 2008 ; pour le débat sur une pré- 

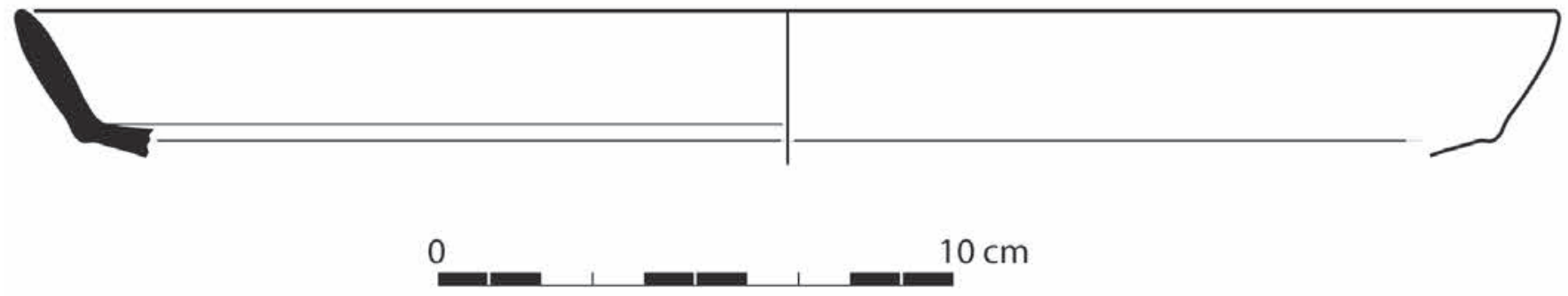

Fig. 8 : Assiette en sigillée italique (DAO F. Cibecchini).

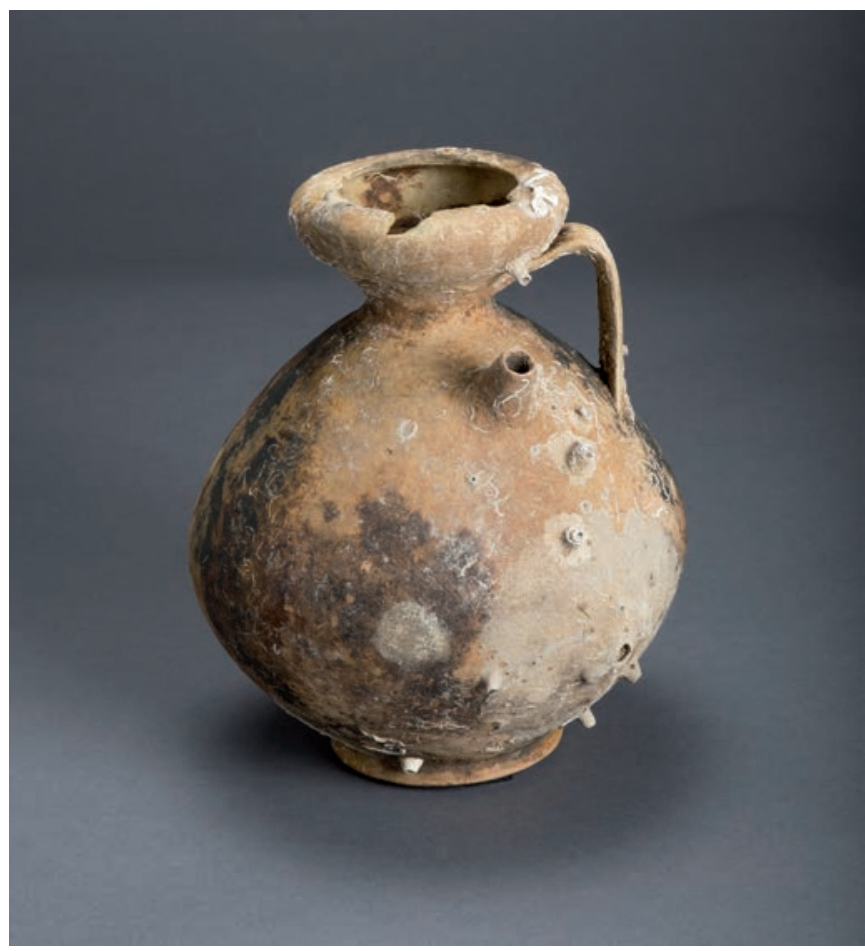

Fig. 9 : Cruche avec filtre et bec verseur, inv. 29115 (cliché T. Seguin).

remplacent dans les zones de production, principalement dans le Picenum et en Cisalpine orientale entre le début de l'époque d'Auguste et le milieu du $\mathrm{I}^{\mathrm{er}}$ s. ap. J.-C.

Il est possible que les deux amphores attribuables à la forme Lamboglia 2, en apparence plus anciennes, fassent partie de la dotation de bord du navire, surtout la L.1. Toutefois la présence des deux autres types, à l'arrière du navire, et l'association avec des amphores de Tarraconaise, nous invitent à une certaine prudence, d'autant que les assemblages sur les épaves montrent souvent la coexistence des formes « classiques » et des formes de transition $^{23}$. En outre, cette association entre les deux types adriatiques, les Oberaden 74 et les Tarraconaise 1 trouve une comparaison assez précise dans l'épave Sud Caveaux 1 où les Lamboglia 2/Dressel 6 sont réutilisées pour le transport de poix ${ }^{24}$.

sumée origine dalmate des Lamboglia 2 et Dressel 6 voir Carre, Monsieur, Pesavento Mattioli 2014

23. Par exemple sur l'épave de Comacchio, datée des années 10 avant J.-C. (Berti 1990).

24. Une comparaison assez précise entre la Lamboglia 2 L. 1 et l'exemplaire dans Long 1998, fig. 3, a ; entre la Lamboglia 2/Dressel 6A L. 3 et Long 1998, fig. 3, d.
Les Lamboglia 2 sont peu diffusées en Méditerranée occidentale et dans le sud de la Gaule, dans les sites terrestres et dans les épaves, où elles sont toujours minoritaires dans les cargaisons tardo-républicaines. Elles sont en revanche bien présentes en Espagne, où plusieurs cargaisons de Lamboglia 2 sont connues, et elles arrivent même à concurrencer les Dressel 1 au sud du Cabo de la Nau, en particulier dans le territoire de Carthagène ${ }^{25}$.

\section{LA VAISSELLE}

La plupart de la vaisselle bien visible dans la zone sud-est, identifiée comme la partie avant du navire, a été récupérée en 2014. Il s'agit d'une cruche avec filtre et bec verseur, un mortier/ pelvis, une grande coupe en céramique commune, un fragment de bord d'une grande assiette en sigillée italique.

Le fragment de lèvre de sigillée italique appartient à une grande assiette de forme Consp. 1.1.3., forme datée entre 40 et 15 avant J.-C. (fig. 8). Ses caractéristiques techniques ${ }^{26}$ laissent planer un doute sur l'origine arétine de cette pièce ${ }^{27}$. L'assiette se trouvait entre les deux jas d'ancre et l'amphore Oberaden 74 fragmentaire à l'extrémité sud-est du site. À proximité, en dessous d'un des jas, se trouvait la cruche en céramique commune avec filtre et bec verseur ${ }^{28}$ (fig. 4, C1 ; fig. 9). Nous n'avons pas trouvé de comparaisons précises pour cette pièce. Deux cruches de ce type, assez proches de la nôtre mais en vernis noir, ont été trouvées sur l'épave d'Anticythère, datée toutefois dans le second quart du II ${ }^{\mathrm{e}}$ s. av. J.-C. (Chidiroglou 2012, p. 197 et p. 207, nn. 281, 282). Elles présentent en particulier le même profil de la lèvre, très rentrant, mais elles sont plus trapues et avec un bec verseur plus long et massif. Leur origine n'a pas été déterminée.

Des cruches avec filtre et bec verseur sont connues en céramique à vernis noir dans de nombreux contextes terrestres de $\mathrm{III}^{\mathrm{e}}-\mathrm{II}^{\mathrm{e}}$ s. av. J.-C. et dans plusieurs autres épaves de l'époque républicaine, datées entre la moitié du $\mathrm{II}^{\mathrm{e}} \mathrm{s}$. et le début du $\mathrm{I}^{\mathrm{er}} \mathrm{s}$. av. J.-C., comme par exemple les épaves Cavalière 1 (Var),

25. Long 1998, p. 343 et 2013, p. 433 pour une liste des épaves ; Molina Vidal 2013, spécialement p. 201 et Pérez Ballester, Pascual Berlanga 2004 pour une vision d'ensemble des dynamiques commerciales liées à la présence des productions adriatiques en Espagne.

26. L'assiette a un diam. max. de $30 \mathrm{~cm}$. La pâte céramique est de couleur noisette, à peine granuleuse, bien épurée et très dure, fracture nette, avec des rares inclusions minuscules blanches. Le vernis, bien conservé à l'intérieur, est de couleur rouge vermillon/corail, épais et bien adhérent.

27. Certes, les modifications de la pâte et du vernis de ce fragment qui empêchent de trancher sur son atelier d'origine, pourraient être dues au long séjour en mer, dans la vase, à grande profondeur. Nous remercions Lucien et Sylvie Rivet pour leur avis sur cette pièce.

28. H. $20 \mathrm{~cm}$; diam. bord $8,8 \mathrm{~cm}$; diam. du fond $8 \mathrm{~cm}$. 

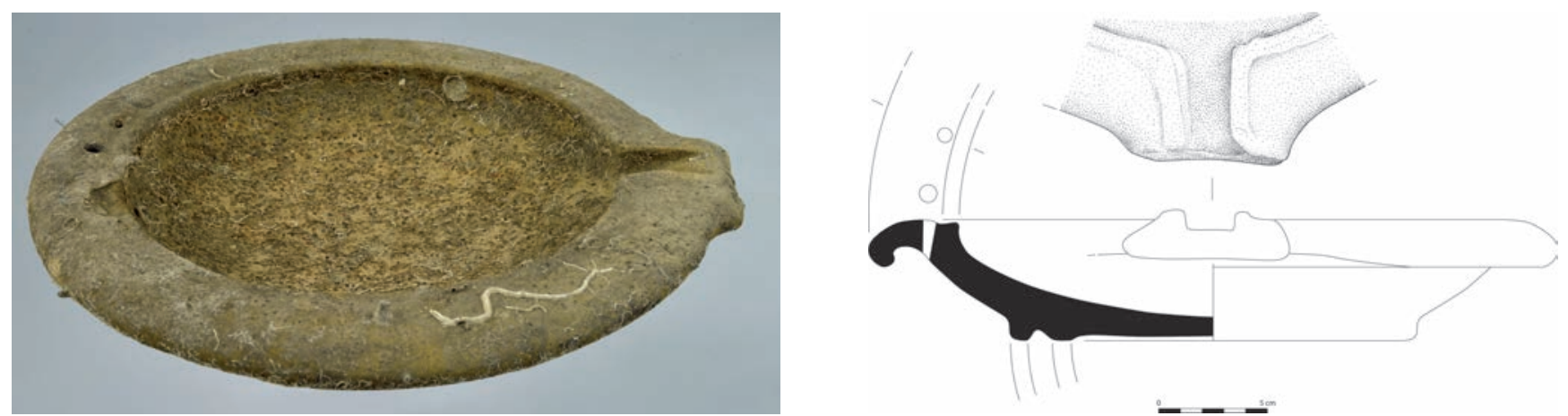

Fig. 10 : Mortier type Dramont D 1, inv. 29113 (prélèvement 2014, dessin M.N. Baudrand, cliché S. Cavillon, Drassm).

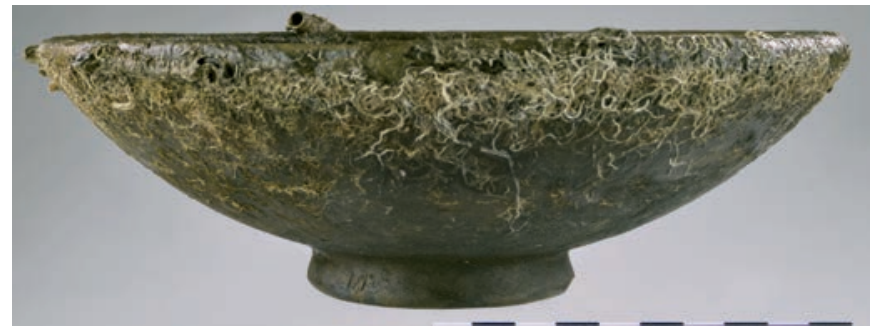

Fig. 11 : Grande coupe en céramique commune, inv. 29114 (cliché S. Cavillon, Drassm).

Sant'Andrea B (Île d'Elbe), Capo Graziano A (Filicudi) ou Escombreras 1 (Carthagène ${ }^{29}$ ).

Le mortier ${ }^{30}$, du type Dramont D 1 (fig. 4, C2 ; fig. 10 A et B), se trouvait en face du bord de l'amphore Lamboglia 2 à l'avant de l'épave ${ }^{31}$. Il se rattache à une production de la côte tyrrhénienne de l'Italie qui a fait l'objet d'un commerce touchant le pourtour méditerranéen mais également dirigé vers le limes rhénan à l'époque augustéenne ${ }^{32}$. Une comparaison assez précise peut être faite avec un mortier attesté à Narbonne (Sanchez 2009a, p. 486, fig. 14, n. 8).

Enfin, n'ayant pu observer la pâte céramique en fracture il est difficile de déterminer la production de la grande coupe en céramique commune ${ }^{33}$ (fig. 4, C3 ; fig. 11) qui se trouvait devant le bord de l'amphore Oberaden 74, toujours à l'avant de l'épave.

La qualité de la couverture photo nous permet d'émettre quelques hypothèses de travail sur des pièces que nous n'avons pas pu encore prélever. Nous avons remarqué deux grands pots à deux anses (fig. 4 et fig. 12, C4 A-B), l'un à côté du tuyau de la pompe de cale sur le côté sud-est et l'autre à quelques mètres de distance vers le nord-ouest, sur les lingots d'étain. Dans le même alignement se trouve un troisième grand pot mais apparemment cassé et sans anses (fig. 4, C4C). Ces pots ont une hauteur d'environ $25 \mathrm{~cm}$ avec

29. Cavalièrel (100-80 av. J.-C.) : Charlin et al. 1978, p. 31, n. 13 et fig. 17, n. 3, fig. 15 , n. 13 ; Ribera 2001, p. 302 et p. 306. Sant'Andrea $B$ (vers 100 av. J.-C.) : Maggiani 1982, p. 73, nn. 59-60. Capo Graziano A (140-120 av. J.-C.) : Cavalier 1985, p. 125-126, fig. 151g, 154a ; Ribera 2001, p. 302. Escombreras 1 (140-130 av. J.-C.) : Scombraria 2004, p. 164 ; Pinedo Reyes, Alonso Campoy 2004, p. 139-144; Cibecchini 2007, tab. 2.

30. H. $6 \mathrm{~cm}$; diam. bord ext. $32 / 33 \mathrm{~cm}$, int. $23 / 24 \mathrm{~cm}$; diam fond ext. $18 \mathrm{~cm}$, int. $11 \mathrm{~cm}$.

31. H. $6 \mathrm{~cm}$; diam. bord ext. $32 / 33 \mathrm{~cm}$, int. $23 / 24 \mathrm{~cm}$; diam. fond ext. $18 \mathrm{~cm}$, int. $11 \mathrm{~cm}$.

32. Pallecchi 2002, p. 42-45, carte diffusion p. 44, fig. 7, Vilvorder dans Brulet, Vilvorder, Delage 2010, p. 372.

33. H. $8 \mathrm{~cm}$; diam. bord $25 \mathrm{~cm}$; diam. fond ext. $10 \mathrm{~cm}$. un diamètre max. d'environ $17-18 \mathrm{~cm}$. Nous n'avons pas trouvé de comparaisons précises mais ce type de conteneur semble rentrer dans une série de pots à deux anses similaires, connus dans des épaves datées entre environ 150 et 50 av. J.-C., comme Cavalière 1, Grand Congloué 2, Sant Jordi A et Titan ${ }^{34}$. Ils ont été rangés dans la céramique commune italique par M. Bats dans le Dicocer, forme COM-IT $2 \mathrm{~b}-2 \mathrm{~d}$, mais leur origine italique n'est pas claire et leur fréquente association dans les épaves avec des céramiques ou des cargaisons provenant de la péninsule Ibérique laisse la porte ouverte à une origine plus occidentale de cette forme. Les pots de Capo Sagro 2 sont plus proches de celui de l'épave du Titan, datée vers 50-45 av. J.-C.

$\mathrm{Au}$ moins trois pièces sont visibles au centre de l'épave, immédiatement au sud de la cargaison de lingots et partiellement mélangés aux amphores. Dans l'axe du navire nous pouvons facilement reconnaître une cruche à une anse (fig. 4, C5 ; fig. 13 et 14), bien visible quoique partiellement ensevelie. Elle pourrait appartenir aux céramiques à pâte grise de la côte catalane, COT-CATCc5, mais encore plus probablement aux cruches en céramique claire récente. Elle trouve plus précisément une bonne comparaison avec la CL-REC 2a, qui domine à Narbonne entre la seconde moitié du $\mathrm{I}^{\mathrm{er}} \mathrm{s}$. av. et la première moitié du Ir s. ap. J.-C. (Sanchez 2009a, p. 481, fig. 15).

Une pièce à l'extrémité est du site (fig. 4, C6) pourrait être identifiée, avec prudence, comme le corps d'un lagynos, ou olpé sans col. Cette hypothèse est renforcée par la présence d'une autre olpé entière (fig. 4, C7 ; fig. 15) à côté d'une des trois Oberaden 74 du côté nord-ouest. Elle pourrait appartenir à une production de céramique claire récente, type CL-REC $2 \mathrm{~g}$, comme celle de Sallèles-d'Aude (A7), bien présente à Narbonne $e^{35}$.

Nous n'avons pas pu identifier d'autres pièces en céramique présentes sur le site car elles sont peu visibles ou trop ensevelies.

34. Épave Grand Congloué 2 (Long 1987, p. 13,Pl. 2, c) datée des années 90-75 av. J.-C. (Ribera 2001, p. 306), Cavalière 1 (Charlin et al. 1978, p. 40, n. 8, fig. 21 et 18, n. 2) et surtout Titan (Taillez 1961, fig. 11) datée autour de 50-45 av. J. C. Dans l'épave Sant Jordi A, datée vers 100-80 av. J.-C. (Ribera 2001, p. 302 et 306), est présente toute une série de cruches à deux anses, de plusieurs dimensions et dont la plus grande a des mesures semblables à celles de Capo Sagro 2, quoique toutes les cruches aient un profil nettement plus cylindrique (Cerdà 1980, p. 51-52, n. 65). Des pots de cette série sont probablement présents aussi sur l'épave Capo Graziano A (Cavalier 1985, fig. 152b, 153, 154a et 155a).

35. Sanchez 2009a, p. 481-484, fig. 12. 

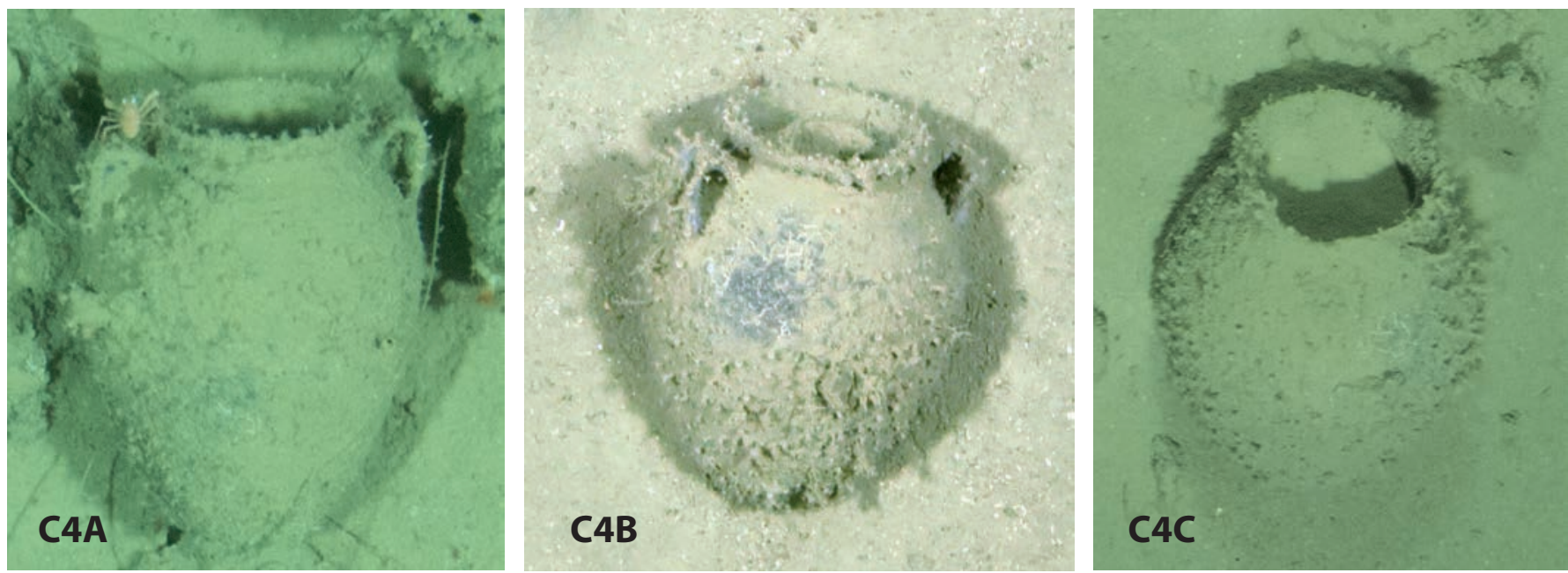

Fig. 12 : Pots à deux anses (C4A-C) in situ (cliché Drassm).

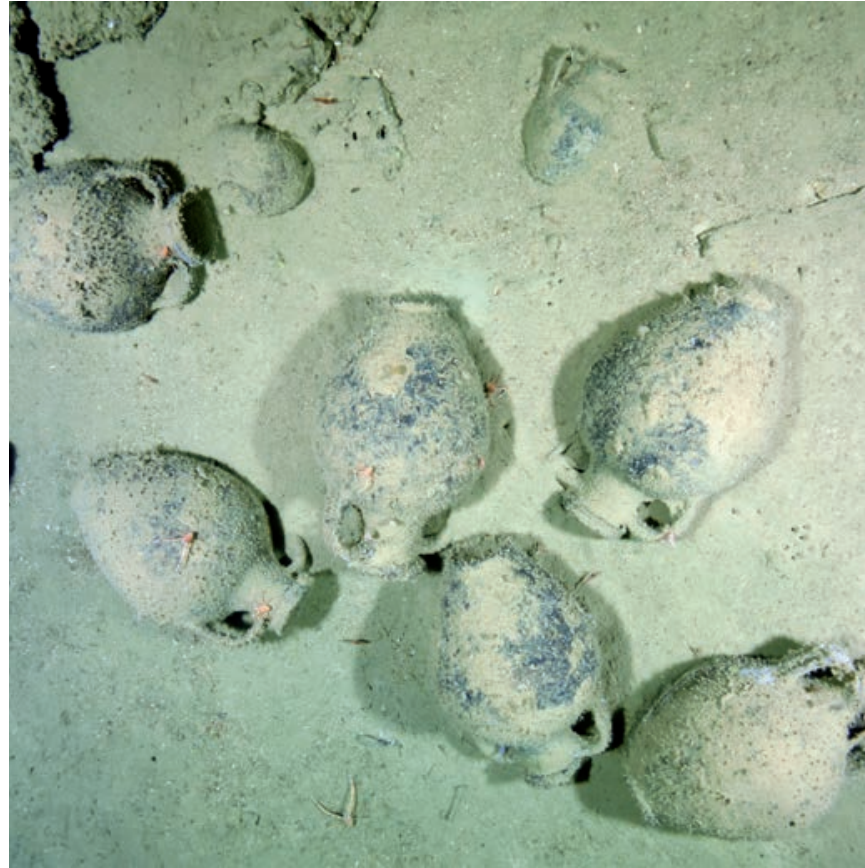

Fig. 13: Olpe à une anse in situ entre les amphores Oberaden 74 au centre du gisement (cliché Drassm).

\section{CONCLUSIONS SUR LE MOBILIER CÉRAMIQUE}

L'ensemble des céramiques fines et les amphores et, plus particulièrement, l'association d'un mortier Dramont D 1, d'un plat Consp. 1.1.3. et des Oberaden 74 permettent de proposer une datation précise entre 20 et 10 av. J.-C. L'association d'amphores Lamboglia 2, Tarraconaise 1 et Oberaden 74, dans des proportions certes fort différentes, rappelle l'assemblage de l'épave SudCaveaux 1 (30-20 av. J.-C.), correspondant à un petit navire qui transportait vraisemblablement de Narbonne vers Marseille une cargaison de poix dans des amphores Lamboglia 2/Dressel 6A et du vin de Léétanie dans des amphores Tarraconaise $1^{36}$. Dans le

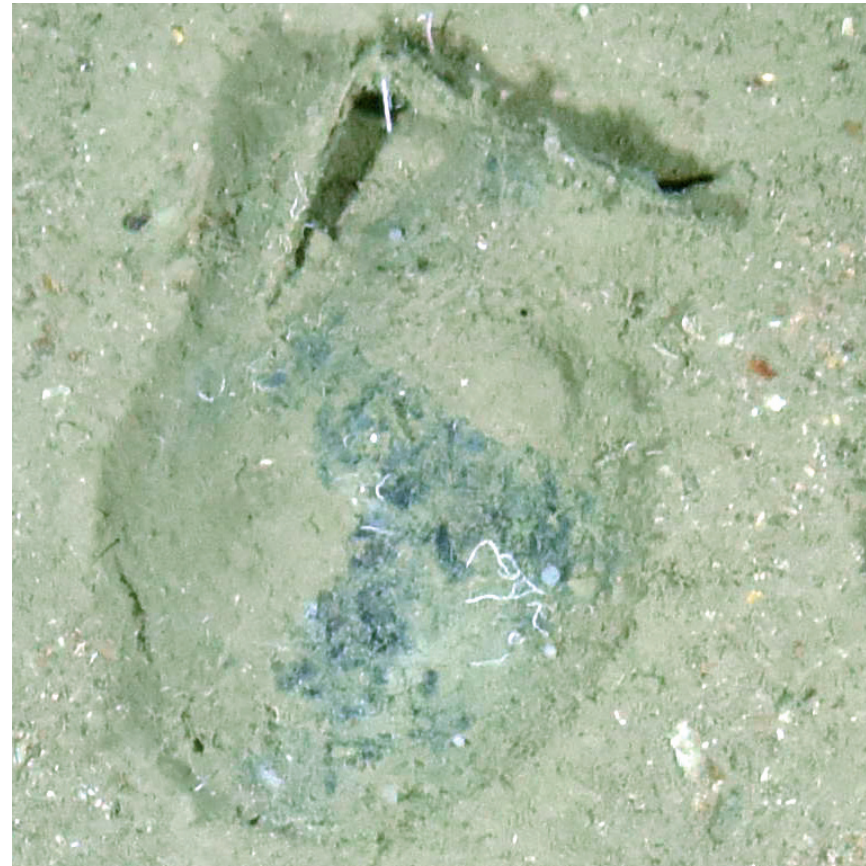

Fig. 14 : Olpe à une anse in situ (cliché Drassm).

cas de Capo Sagro 2, Narbonne apparaît comme le principal point commun entre ces céramiques, et en particulier les amphores. Port de stockage et de redistribution le plus important de la région, surtout pour le vin, son rôle a ainsi été particulièrement mis en évidence par les auteurs anciens (Sanchez 2009b, p. 261). C'est ce que confirment des recherches récentes, qui insistent sur les relations privilégiées, dès le milieu du $\mathrm{I}^{\mathrm{er}} \mathrm{s}$. av. J.-C., entre Narbonne et la Tarraconaise, essentiellement d'ordre commercial mais aussi liées à l'élite politique et aux intérêts économiques des grandes familles narbonnaises en Catalogne ${ }^{37}$. 


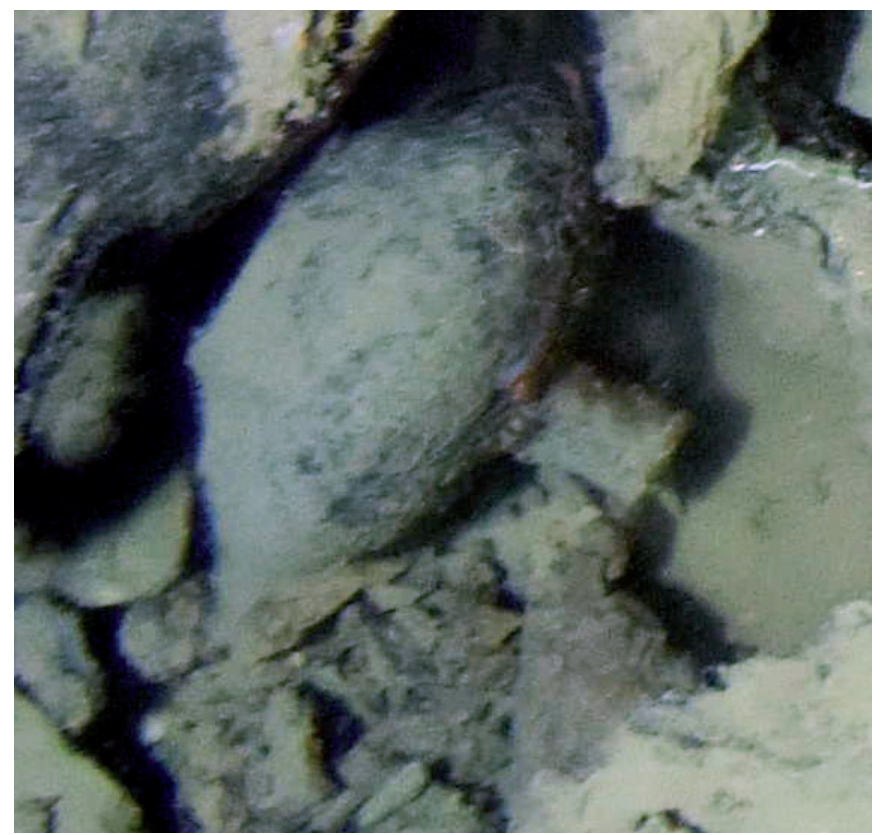

Fig. 15 : Olpe/lagynos in situ (cliché Drassm).

\section{CAPO SAGRO 2 : UN NOUVEAU JALON SUR LES ROUTES DU COMMERCE ROMAIN DE L'ÉTAIN EN MÉDITERRANÉE}

L'étain est un métal rare et il fut, pour cette raison, un métal essentiel dans l'Antiquité, indispensable au travail des bronziers $^{38}$. On ne le trouve quasiment pas dans le monde méditerranéen, et les rares gisements qui y sont attestés, ainsi dans la Sierra de Cartagena, intensément exploitée pour ses minéralisations argentifères à la fin de la République, n'ont pas fourni de vestiges trahissant une mise en valeur de quelque envergure à l'époque romaine. Les plus importants gisements d'étain en Occident sont inclus dans un large arc de cercle qui relie les îles Britanniques à l'Estrémadure espagnole englobant ainsi la Cornouailles anglaise, l'Armorique, la Galice et le nord du Portugal (fig. 16) (Domergue 2008, p. 89-90). L'archéologie n’a pas encore identifié d'exploitations stannifères antiques, en raison de conditions de gisements tout à fait particulières. Le principal minerai d'étain, la cassitérite, se trouve en effet dans des dépôts alluviaux et des placers fluviaux : l'extraction se faisait donc à ciel ouvert et les traces qu'elle dut laisser ont été bien souvent masquées, quand elles n'ont pas été détruites, lors de la reprise de l'exploitation de ces dépôts à des époques plus récentes. Quant au minerai en roche, notre ignorance est totale tant sur l'emplacement des gisements que sur les conditions de leur exploitation dans l'Antiquité. Il est donc aujourd'hui impossible de dire quelles furent les régions qui comptèrent à l'époque romaine dans l'approvisionnement en étain des régions méditerranéennes. Or l'étain circulait bien sur les routes maritimes de la Méditerranée mais les données restent encore très partielles sur son ou ses origines. D'une part, l'étain était

38. On rappellera pour mémoire l'anecdote transmise par Strabon, 3, 5, 11, sur les manœuvres déployées par les négociants de Gades pour tenir secrète face aux Romains la route menant aux célèbres et mythiques Îles Cassitérides qui, selon la tradition, regorgeaient de minerai d'étain. commercialisé en lingots dont la typologie variée ne permet pas de déterminer la provenance. D'autre part, les cargaisons auxquelles appartiennent ces mêmes lingots sont généralement insuffisamment connues pour pouvoir, là encore, leur attribuer une origine géographique précise. Il faut prendre en compte aussi le fait que certaines cargaisons, comme ce fut vraisemblablement le cas pour Capo Sagro 2, ont été recomposées dans des ports qui ne furent pas nécessairement les débouchés maritimes des zones minières concernées, ce qui ne facilite pas, on l'aura compris, l'attribution des lingots à un secteur minier précis. Enfin, à l'inverse du plomb, du cuivre mais aussi du fer, on ne dispose aujourd'hui d'aucune méthode archéométrique éprouvée qui permette de cibler l'origine du métal. Le plomb ne s'y trouve qu'en quantités infimes, rendant inopérantes les analyses isotopiques du plomb (LIA, Lead Isotopic Analyses) qui ont montré au contraire leur efficacité pour le cuivre et, évidemment, le plomb ${ }^{39}$.

Le nombre d'épaves renfermant des lingots d'étain reste aujourd'hui réduit, s'y ajoutent quelques découvertes isolées (fig. 16 ; tableau 1).

La plus ancienne épave à chargement de lingots d'étain est Bagaud 2, dans les îles d'Hyères. Datée de la fin du $\mathrm{II}^{\mathrm{e}} \mathrm{s}$. - début du I ${ }^{\text {er }}$ s. av. J.-C., elle renfermait peut-être à l'origine une centaine de lingots, dont 45 en forme de galettes planoconvexes et de troncs de pyramide plus ou moins réguliers, d'un poids moyen de $25 \mathrm{~kg}$, ont pu être remontés ${ }^{40}$. Dans le même secteur, à Porquerolles, l'épave Mèdes 1 ( $\mathrm{I}^{\mathrm{er}} \mathrm{S}$. av. J.-C.), renfermant également des barres de fer, a livré deux saumons d'étain, allongés et aux extrémités arrondies (Tchernia 1969, p. 476-478; Sabastia à paraître). De la même époque probablement datent les quelques lingots plano-convexes remontés d'une épave récemment identifiée au large des Saintes-Mariesde-la-Mer (SM17) (Long 2015, p. 60). Port-Vendres II, épave d'époque claudienne, a livré dans une cargaison dominée par les amphores du sud de l'Espagne, pas moins de quarante-sept lingots ${ }^{41}$ d'une forme particulière, du type «bourse » ou «sac à main ». Des lingots de ce type sont également connus sur la côte orientale de la Sardaigne, dans l'épave de Capo Bellavista ou Arbatax (Lo Schiavo 1986, p. 135-138), en Corse sur le site de Plage de Losari (Bernard 2003, p. 59-60), ou encore à Ischia (deux exemplaires ${ }^{42}$ ), et en un exemplaire enfin sur l'épave Lavezzi 2 (Parker 1992, n 585). Face à l'Italie, à Ventotene, l'épave Cala Rossano, malheureusement pillée, a livré une quinzaine de petits lingots en forme de pain de sucre bombés pourvus d'un appendice facilitant leur manutention, le reste de la cargaison étant composé d'amphores à saumures de Bétique. Un lingot isolé, d'un poids de $10,3 \mathrm{~kg}$, a été d'autre part repêché en 2010 par des plongeurs sportifs au large de Porto Ferro à Nurra (Sassari); de forme tronco-pyramidale, il est pourvu d'une grande anse arrondie et de deux ergots laté$\operatorname{rau}^{43}$. Ne figure pas enfin dans le tableau une épave de

39. Voir par ex., Klein et al. 2007 (cuivre) ; Trincherini et al. 2009 (plomb de Carthagène) ; Domergue et al. 2012 (plomb de Sierra Morena).

40. Long 1985, p. 93-98; id., 1987, p. 151. Le poids total pourrait donc avoir approché les deux tonnes et demie.

41. Cf. Colls et al. 1975, p. 61-94; 1977, p. 11-18. Vingt-neuf sont inédits. Étudiés au dépôt archéologique de Port-Vendres en mai 2002 par Claude Domergue et Ch. Rico.

42. Inédits. Conservés à la Villa Arbusto à Ischia (nº 227926 et 227 927).

43. M. Galasso, Il lingotto di stagno di Porto Ferro (Sassari), notizia preliminare:

https://www.academia.edu/9331536/Il_lingotto_di_stagno_di_Porto_Ferro_ Sassari_-_di_Mario_Galasso 


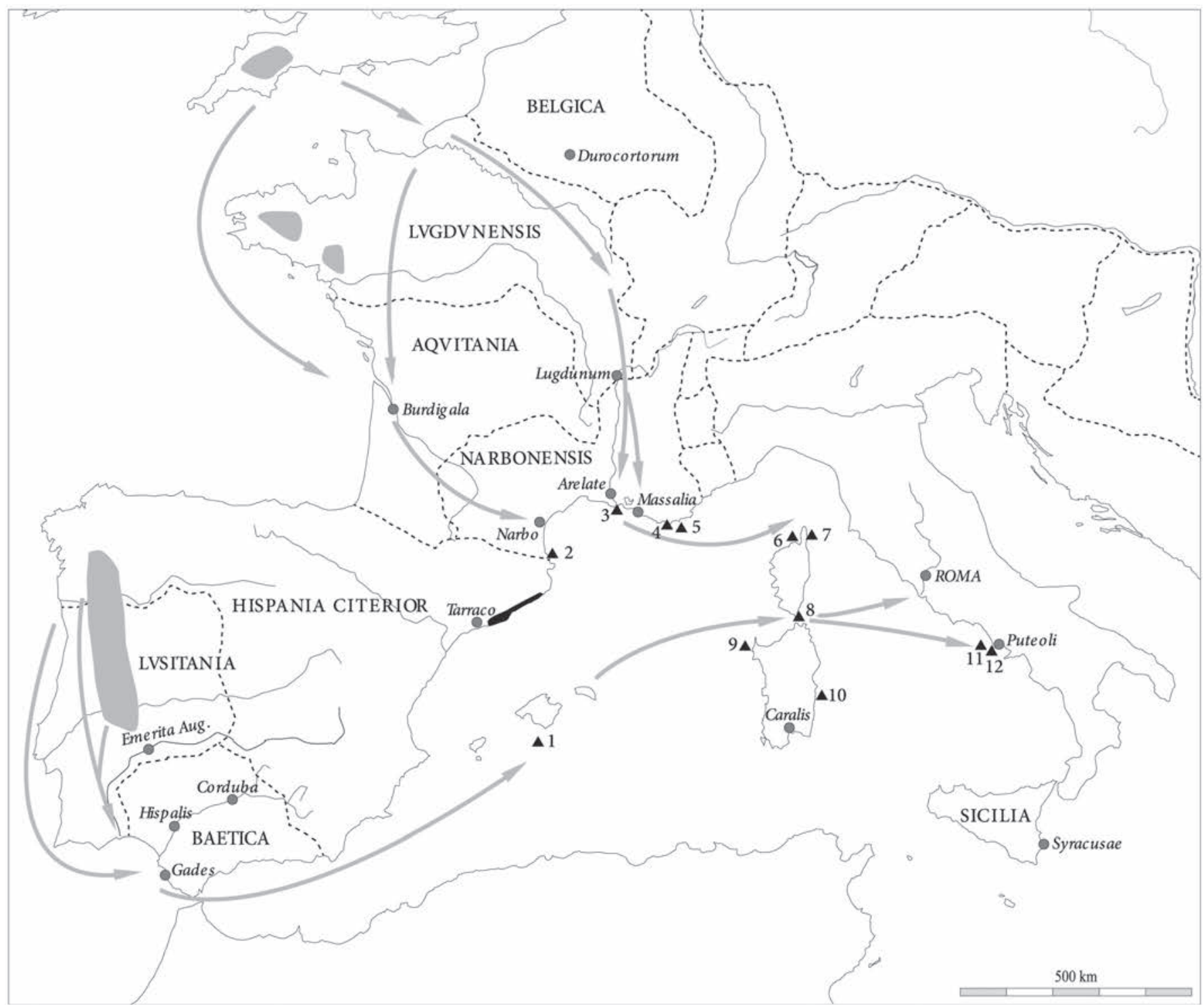

Fig. 16 : L'étain en Méditerranée et Europe occidentale (élaboration C. Rico). Zones en gris : principaux districts stannifères exploités dans l'Antiquité ; zone en noir : région de production des amphores Oberaden 74 et Tarraconaise 1 ; flèches : grands itinéraires supposés de l'étain à l'époque romaine ; triangles : épaves et gisements sous-marins ayant livré des lingots d'étain : 1- Na Redona ; 2-Port-Vendres II ; 3- Saintes-Maries-de-la-Mer 27 ; 4- Bagaud 2 ; 5- Mèdes 1 ; 6- Plage de Lozari ; 7-Capo Sagro 2 ; 8 - Lavezzi 2 ; 9 -Porto Ferro ; 10-Capo Bellavista ; 11 -Cala Rossano ; 12-Ischia.

Tableau 1 : Tableau de synthèse des épaves antiques chargées d'étain.

\begin{tabular}{|c|c|c|c|c|}
\hline & Nature du site & Nombre & Autres produits & Date \\
\hline Bagaud 2 & Épave & 45 (remontés) & Barres de fer & $1^{\text {re }} \mathrm{m} . \mathrm{I}^{\mathrm{er}} \mathrm{s}$. av. J.-C. \\
\hline Mèdes 1 & Épave & 2 & Barres de fer & $\mathrm{I}^{\mathrm{er}} \mathrm{s} . \mathrm{av} . \mathrm{J} .-\mathrm{C}$. (?) \\
\hline Capo Bellavista & Épave & 32 (6 conservés) & Barres de fer & Ier s. ap. J.-C. (?) \\
\hline Port-Vendres II & Épave & 47 & a. Bétique ; plomb et cuivre & Claude \\
\hline Lavezzi 2 & Épave & 1 & a. Bétique & Milieu Ir s. ap. J.-C. \\
\hline Cala Rossano & Épave & 15 & a. Bétique (salaisons) & 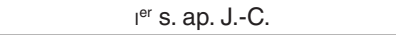 \\
\hline Plage de Lozari & Épave & 5 & a. Bétique (Dr. 20) & I $^{\text {er }}$ s. ap. J.-C. (?) \\
\hline Ischia & Trouvaille isolée & 2 & - & - \\
\hline Porto Ferro & Trouvaille isolée & 1 & - & Romain? \\
\hline Saintes-Maries-de-la-Mer 17 & Épave & 7 (remontés) & - & Fin de la République - Haut-Empire \\
\hline
\end{tabular}

découverte ancienne à Cabrera dans les Baléares, Na Redona, pillée au début des années 1960. Les informations sont succinctes sur cette épave et même contradictoires. Elle aurait renfermé plusieurs centaines d'amphores à saumures et un nombre indéterminé de lingots de cuivre. Parker lui attribue plus de 400 lingots d'étain en forme de demi-sphère et d'un poids à l'unité de $10 \mathrm{~kg}$ (Parker 1992, n 980). De leur côté, Veny et Cerdà (1972, p. 299), reprenant une information orale de J. Mascaró Pasarius, font allusion à des « panes de estaño en forma de media naranja y de unos $60 \mathrm{~kg}$ de peso », mais 
sans en donner le nombre. Il est difficile de dire s'il s'agit du même site.

On le voit, les informations sont partielles sur les épaves et leurs chargements, et n'aident pas à déterminer l'origine du métal et à reconstituer les itinéraires suivis par l'étain en Méditerranée romaine. La péninsule Ibérique, une des principales régions pourvoyeuses de métaux, nobles et vils, dans l'Antiquité, est souvent privilégiée comme lieu d'origine de l'étain. Il en irait ainsi des lingots de l'épave Cala Rossano, au vu des restes d'amphores à saumures qu'elle renfermait ${ }^{44}$. Les cinq lingots de l'épave Plage de Lozari en Haute-Corse pourraient avoir la même origine, si les fragments d'amphores Dr. 20 découverts sur le site appartiennent à la même épave. Les lingots de Port-Vendres II, que tout dans le chargement renvoie au sud de l'Espagne, sont traditionnellement donnés comme hispaniques. En revanche, rien ne permet d'attribuer une origine à l'étain des épaves Capo Bellavista, Mèdes 1 et Bagaud 2. L'épigraphie de son côté ne fournit aucune piste. Les inscriptions ne manquent pourtant pas et même foisonnent, c'est le cas des lingots de Port-Vendres II et de Plage de Lozari. Elles mentionnent des individus dont l'intervention dans le processus de fabrication ou dans le commerce n'est pas assurée. Elles ne sont en tout cas d'aucun secours pour déterminer l'origine des lingots.

Comme on l'a dit, l'étain est présent sur toute la façade atlantique de l'Europe, et notamment en Gaule (Bretagne actuelle) et dans le sud de la Grande-Bretagne, en Cornouailles. L'intérêt pour le métal blanc de ces régions, et de la seconde en particulier, semble s'être manifesté assez tôt si l'on en croit Diodore de Sicile (V, 22). Celui-ci, reprenant Posidonius, voire une information plus ancienne encore, révèle comment l'étain de Cornouailles était expédié vers l'embouchure du Rhône dans un long voyage qui ne durait pas moins de trente jours à travers la Gaule $^{45}$. À l'époque romaine, et à l'époque impériale plus particulièrement, le plomb germanique mais aussi celui produit en Britannia parvenaient jusqu'en Méditerranée ${ }^{46}$. En alla-t-il de même avec l'étain ? La question a été posée à propos des lingots de Port-Vendres II, dont l'origine hispanique semble pourtant aller de soi si on tient compte du reste de la cargaison à laquelle ils appartenaient (Colls et al. 1975, p. 84). C'est d'abord C. Roden qui a argumenté en faveur d'une provenance bretonne, s'appuyant sur la découverte ancienne de deux lingots d'une typologie proche des lingots de Port-Vendres au cœur de la principale zone stannifère anglaise des Cornouailles, dans le secteur de St Austell Moors à Petenwan Valley ${ }^{47}$. La « piste bretonne » a pris récemment davantage de force après l'étude, réalisée en 2002, de plusieurs lingots de la même épave non encore publiés (Rico 2011, p. 59-63). L'hypothèse a été défendue alors d'un commerce de redistribution à partir de Narbonne que les lingots auraient pu atteindre en suivant un itinéraire un peu différent de celui évoqué par Diodore. Il aurait emprunté le célèbre

44. Le navire de $\mathrm{Na}$ Redona, assurément, serait parti de quelque port du sud de l'Hispanie, ce qui a priori militerait en faveur d'une origine hispanique pour les lingots d'étain qui composaient sa cargaison.

45. Voir l'étude récente de Mairécolas, Pailler 2010, p. 145-160 qui, à partir des textes, des données de l'archéologie, de la toponymie et de l'ethnonymie, restitue les probables «voies de l'étain » qui permettaient depuis l'âge du Bronze de faire parvenir l'étain breton, et, peut-être aussi gaulois, jusqu'en Méditerranée.

46. Épaves $S M I$ en Camargue et Rena Maiore en Sardaigne (plomb germanique) ; lingots de Fos (pb breton). Voir, en dernier lieu, Domergue, Rico, à paraître.

47. Roden 1985 , p. 54-55. Leur poids est cependant plus important, 13,5 kg contre $8,520 \mathrm{~kg}$ pour le plus lourd des lingots de Port-Vendres II.
« isthme gaulois » qui, par la vallée de la Garonne et celle de l'Aude, permettait aussi de rejoindre la Méditerranée à Narbonne, emporion que le même Diodore présente plus loin dans son récit $(\mathrm{V}, 38)$ comme l'autre débouché méditerranéen de l'étain breton. La même provenance peut être aussi envisagée pour l'étain de Bagaud 2 et de Mèdes 1. On tiendra compte en effet de la présence, dans l'une et l'autre épave, de barres de fer, qui pourrait trahir des cargaisons recomposées. Certes la provenance de ce fer n'est pas connue, mais à l'époque des deux naufrages, les $\mathrm{II}^{\mathrm{e}}$ et $\mathrm{I}^{\mathrm{er}} \mathrm{s}$. av. J.-C., on ne voit guère que la Gaule comme lieu d'origine probable de ce métal. On peut dès lors se demander si, à l'instar de Port-Vendres II, ces navires n'avaient pas été chargés dans un port du sud de la Gaule. La position de l'une et l'autre épave dans les îles d'Hyères serait un élément dans ce sens (Domergue, Rico, à paraître). Les deux navires seraient partis d'un port du sud de la Gaule, par exemple Marseille, et faisaient route vers l'Italie quand ils ont sombré. Les timbres en caractères grecs que portent les lingots de Bagaud 2 trouveraient ici toute leur signification (Long 1987, 152-155). Ils mentionnent un certain Herakleides dans lequel on verra le négociant ayant acquis les lingots, peut-être donc à Marseille; il les frappe alors d'un cachet à son nom ajoutant le vocable d'Hypokeltoi pour certifier l'origine du métal. L'appellation «Celtes d'en bas » ou « du sud » reste cependant énigmatique. Désigne-t-elle des Ibères ? Des Bretons ? Il est difficile de trancher même si notre préférence irait aux seconds.

Quoi qu'il en soit, on peut raisonnablement envisager aujourd'hui que de l'étain de provenances diverses, principalement hispanique et bretonne, circulait régulièrement en Méditerranée occidentale à l'époque de la domination romaine (fig. 16). Deux grands axes de circulation existaient ; l'un, au départ de la péninsule Ibérique, utilisait sans nul doute les mêmes itinéraires que le plomb et le cuivre hispaniques ${ }^{48}$. Le second, emprunté quant à lui par l'étain de Cornouailles, traversait la Gaule du nord au sud pour aboutir aux grands ports méditerranéens, Narbonne, peut-être Arles-Fos ou encore Marseille. L'étain était alors redistribué par voie maritime avec d'autres marchandises de provenances diverses, locale ou extérieure, entreposées dans ces mêmes ports. Ce fut visiblement le cas aussi de l'étain de Capo Sagro 2.

\section{ESTIMATION DES DIMENSIONS ET DU TONNAGE DU NAVIRE}

La question de comment établir les dimensions et calculer le tonnage théorique d'un navire à partir de l'observation des vestiges visibles d'une épave située à grande profondeur compte parmi les interrogations que se posent de plus en plus souvent les archéologues navals en raison de la multiplication des prospections profondes ${ }^{49}$. Dans la plupart des cas, l'exceptionnelle conservation de la cargaison permet, par simple comptage des éléments qui composent la couche superficielle, d'effectuer une

48. Voir en dernier lieu Domergue, Rico 2014, p. 147-151.

49. On peut citer parmi les quelques exemples les plus récents : les épaves de Tanit et Elissa, au large d'Israël, datées du viII ${ }^{\mathrm{e}}$ s. av. J.-C. (Ballard et al. 2002, p. 151-168) ou bien encore l'épave maltaise de Xlendi, datée du début du VII ${ }^{\mathrm{e}} \mathrm{s}$. av. J.-C. (Sourisseau 2015, consulté en ligne le 10/11/2016 :

http://www.lsis.org/groplan/papers/groplan_livrableXlendiCargaisonOctobre2015.pdf). 


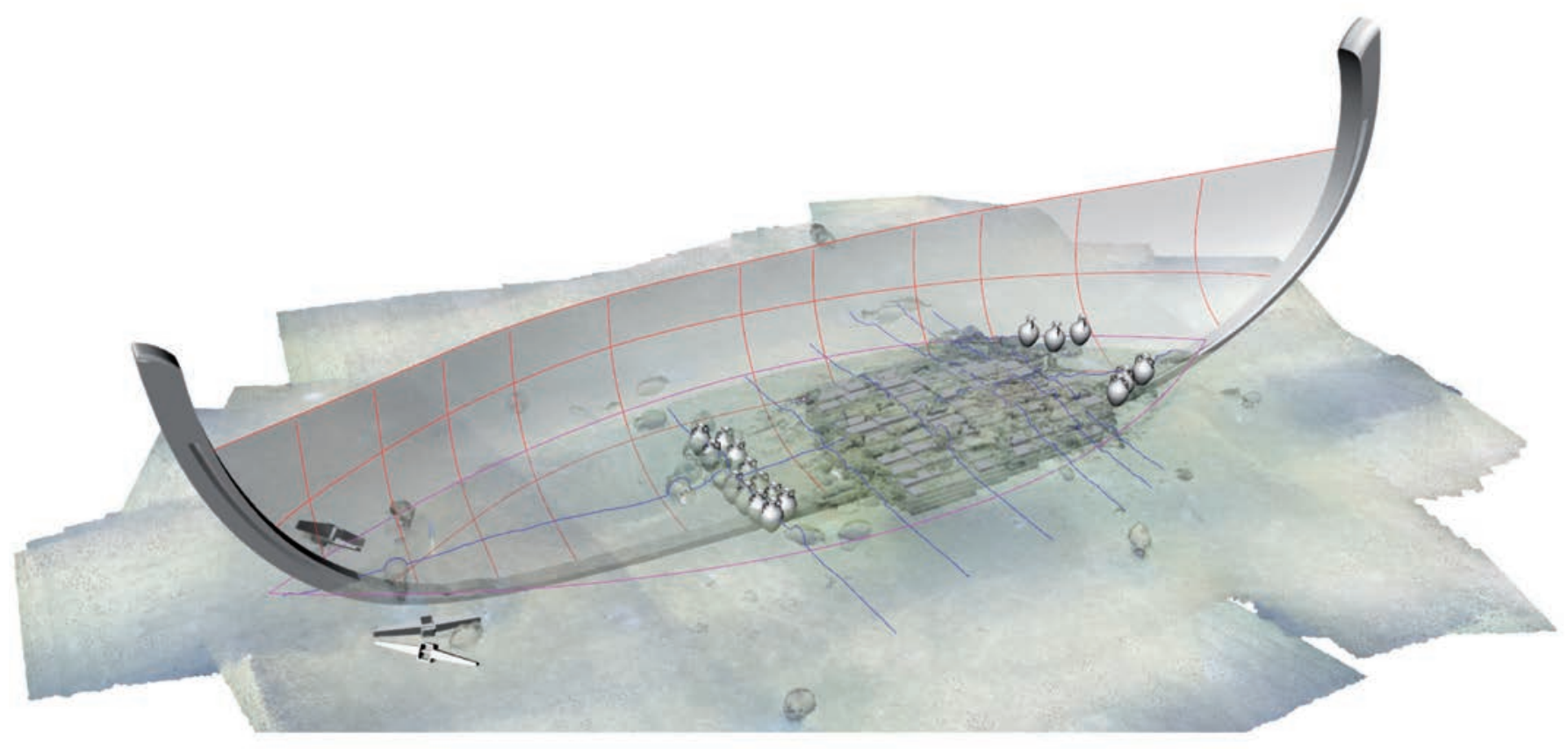

P. Poveda, AMU-CNRS, CCJ, 2017

Fig. 17: Vue du modèle 3D d'étude de restitution de la cargaison (élaboration P. Poveda).

estimation relativement proche de la réalité du poids original théorique de toute la cargaison. Toutefois l'imprécision de cette méthode provient naturellement de la présence d'importantes parties de la cargaison enfouies dont il est difficile d'estimer les proportions et de connaître la nature, faute de pouvoir pratiquer une véritable fouille à de telles profondeurs. Par ailleurs, ce comptage des parties visibles ne permet de calculer que le chargement du navire au moment de son naufrage ${ }^{50}$. Cependant, si le comptage est associé à l'appréciation des dimensions globales du gisement, il est possible d'établir un ordre de grandeur du tonnage de ces navires qui paraît globalement satisfaisant et, enfin, assez proche de la réalité.

La photogrammétrie très précise réalisée en 2015 permet ainsi d'évaluer les dimensions et le tonnage théorique d'origine du navire Capo Sagro 2. Certes le procédé d'évaluation du poids de la cargaison de l'épave présente les inconvénients déjà soulignés. Malgré le fait qu'une grande partie du gisement soit enfoui, le grand nombre de données morphologiques identifiables permet de préciser l'interprétation du gisement et de proposer une estimation plus fine des dimensions et du tonnage du navire (fig. 4 et 17).

En effet, au-delà de la simple donnée fournie par l'emprise totale de la cargaison (amphores et lingots), on distingue sur le gisement des marqueurs significatifs de la morphologie originale du navire. À l'extrémité sud-est tout d'abord, on remarque la présence de quatre jas d'ancre qui paraissent signaler l'une des limites du navire. À l'opposé, au nord-ouest du tumulus, les vestiges de la cargaison marquent très nettement la fin du gisement. On distingue aussi sur les bords du tumulus, formé par les lingots d'étain, l'affleurement ponctuel du bordé et des têtes de membrures. Or ces éléments présentent dans le plan une courbure bien symétrique sur les deux bords de l'épave, ce qui

50. Sur ces problèmes voir Pomey, Rieth 2005, p. 43-44. autorise à en restituer les formes à cet endroit précis, formes qui dénotent clairement ici la fermeture de la carène. Entre ces deux extrémités, l'axe longitudinal apparaît bien marqué au sein de la cargaison, comme si le chargement restituait par-là dans sa forme la présence d'un obstacle d'importance tel qu'une carlingue ou une emplanture. Enfin, dans la partie nord-ouest du chargement, la présence de canalisations en plomb semble marquer la position de la pompe de cale. Traditionnellement placée à l'aplomb de la partie la plus basse de la coque, on peut raisonnablement la restituer sur les navires de commerce de cette époque entre le quart et le tiers arrière du bateau. Ces canalisations s'accompagnent par ailleurs, sur l'axe longitudinal du gisement, d'une dépression carrée qui marque l'emprise d'une structure que l'on peut interpréter comme la cage de protection de la pompe, à l'image de ce qui a pu être mis en évidence sur l'épave de la Madrague de Giens (Pomey 1982, p. 138) ou encore sur celle de l'épave Dramont E (Santamaria 1995, p. 171-174).

L'ensemble de ces données permet de définir déjà plusieurs éléments d'importance concernant le navire d'origine : d'une longueur minimale de $16,20 \mathrm{~m}$ pour une largeur de $5 \mathrm{~m}$, son étrave peut vraisemblablement être restituée à l'extrémité sud-est du gisement à l'endroit où reposent les quatre jas d'ancre en plomb. Par ailleurs, compte tenu de la dégradation des œuvres mortes et de la position des éléments les plus épars tels que les tuyaux en plomb et les jas d'ancre, on peut envisager de restituer les dimensions du navire à 20,50 m environ pour une largeur de $7 \mathrm{~m}$ (fig. 4).

Pour le tonnage théorique, si l'on considère un navire de 18 à $22 \mathrm{~m}$ de longueur pour une largeur de 6 à $7 \mathrm{~m}$, il est possible d'établir, à partir d'une rapide étude tridimensionnelle, un déplacement de l'ordre de 100 à 120 tonnes métriques, ce qui correspondrait à un port en lourd (charge utile) de 70 à 90 tonnes. De la même manière, la morphologie du tumulus dans la zone de chargement des lingots permet aussi d'établir une hauteur minimale d'empilement de $40 \mathrm{~cm}$ correspondant à la hauteur moyenne du chargement de lingots mesurée depuis le niveau du fond de cale. 


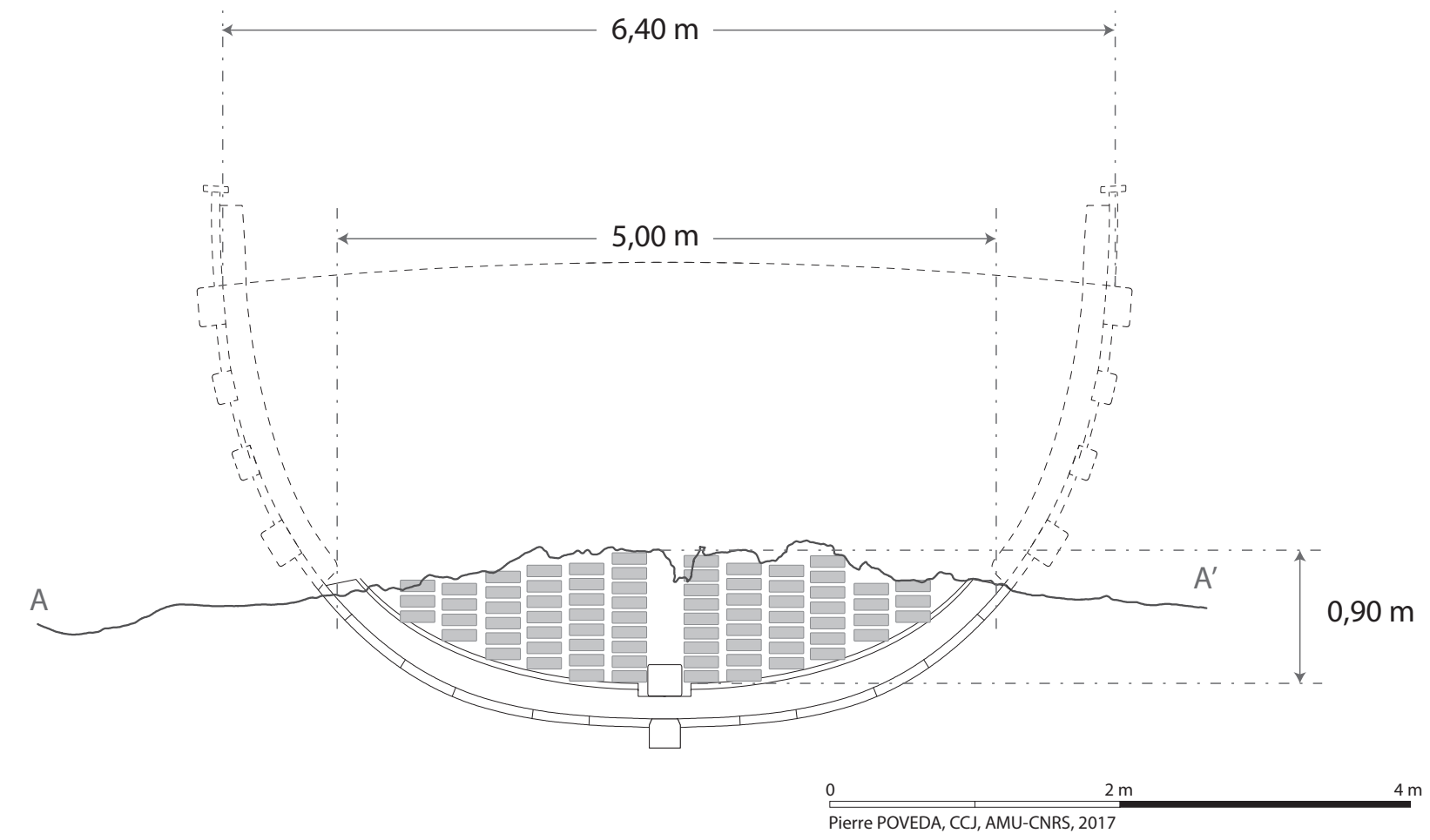

Fig. 18: Restitution schématique de la cargaison au niveau de la section Transversale A-A' (élaboration P. Poveda).

Si l'on ajoute à cette donnée le nombre de 84 lingots visibles sur la couche supérieure, ainsi que leur apparente disposition, nous pouvons proposer une évaluation de la cargaison d'étain. Toutefois, compte tenu de certaines inconnues tel que le nombre décroissant de lingots par couche dans les parties inferieures, leur empilement en assises croisées ainsi que leur grande hétérogénéité typologique, on ne peut que rappeler le caractère tout à fait approximatif de cette démarche. Ainsi, nous pouvons estimer le volume occupé par la cargaison d'étain à une quinzaine de mètres cubes $\left(16,6 \mathrm{~m}^{3}\right)$ correspondant à l'espace inférieur de la cale, contenu entre la pompe de cale d'une part et le pied de mât d'autre part. Ce volume, en tenant compte d'un coefficient d'arrimage relativement faible de $0,39 \mathrm{~m}^{3} / \mathrm{t}^{51}$, permet de restituer une cargaison homogène d'environ 1100 lingots médians (semblables à ceux du type 1 décrits précédemment : $60 \mathrm{~cm}$ de longueur pour $22 \mathrm{~cm}$ de largeur et $5 \mathrm{~cm}$ d'épaisseur) (fig. 18). Cette estimation correspondrait à un poids total pour cette partie de la cargaison d'environ 42,5 tonnes, pour des lingots d'un poids unitaire de $40 \mathrm{~kg}$ en moyenne ${ }^{52}$.

\section{CONCLUSION}

Les contraintes posées par la très grande profondeur de l'épave empêchent de réaliser une fouille du gisement et donc d'avoir une idée précise de son chargement de métal. D'après les observations effectuées et les mesures très précises que l'exploration superficielle a permis de réaliser, la taille du chargement a été établie à un millier de lingots pour un poids maximum

51. Cette valeur correspond à une densité de la cargaison de lingots de 2,55 $\mathrm{t} / \mathrm{m}^{3}$. Elle doit être mise en parallèle avec la masse volumique de l'étain brut de $5,75 \mathrm{t} / \mathrm{m}^{3}$.

52. Poids moyen calculé d'après la densité de l'étain qui est de $5,75 \mathrm{~g} / \mathrm{cm}^{3}$. autour de 40 tonnes. Cela paraît considérable, et Capo Sagro 2 se hisserait à la première place des épaves connues renfermant un chargement métallique, devant Mal di Ventre $C$ (Sardaigne occidentale) et ses mille lingots de plomb de Carthagène représentant un chargement proche de 30-35 tonnes (Salvi 1992). Plus gros chargement d'étain connu aujourd'hui en Méditerranée romaine ${ }^{53}$, l'épave Capo Sagro 2 illustre l'existence d'un trafic maritime régulier de l'étain, au même titre que le plomb et le cuivre. En ce sens, elle se distingue de Port-Vendres II dont les 47 lingots d'étain récupérés paraissent davantage correspondre à une commande spécifique (Rico 2011, p. 63). Il est donc vraisemblable que l'étain ait constitué, sinon la cargaison principale du navire, du moins la marchandise à plus forte valeur ajoutée. Si l'on en croit Pline l'Ancien (NH, XXXIV, 161), l'étain se négociait au prix de 80 deniers la livre. Le calcul est rapidement fait : la cargaison d'étain de Capo Sagro 2 représenterait une valeur de 9600000 deniers, un chiffre qui donne le tournis ! Et qui pose bien des questions sur l'identité tant des commerçants qui l'avaient réunie que de son ou ses destinataires.

On peut supposer que celle-ci était destinée à quelque marché de l'Italie, Rome, voire Pouzzoles, ce que semble a priori confirmer la localisation du naufrage. Si l'on ignore tout des circonstances de l'accident, le naufrage survint une fois que le navire avait doublé le Cap Corse. Le point de départ du voyage devait donc être la péninsule Ibérique ou la Gaule. En apparence, militent en faveur de la première hypothèse la plupart des amphores identifiées sur le site. Elles sont toutes de fabrication hispanique ; en l'occurrence elles proviennent des ateliers du nord de la province de Tarraconaise. Pour autant, les lingots, de leur côté, ne peuvent avoir la même origine, aucune exploitation d'étain n'étant attestée dans le nord de la Péninsule. D'après la

53. Cette cargaison n'est comparable qu'à celle récemment découverte sur l'épave Bloscon 1 à Roscoff en Bretagne, toutefois bien plus tardive, datée entre le $\mathrm{III}^{\mathrm{e}}$ et le $\mathrm{IV}^{\mathrm{e}}$ s. ap. J.-C. : Hulot 2017. 
disposition des vestiges de l'épave, il apparaît que le chargement du bateau s'est fait en une seule fois, les lingots ayant pris place avant les amphores et d'autres marchandises éventuelles, de nature périssable, qui ne seraient pas parvenues jusqu'à nous. Mais il est vrai que la valeur de la cargaison d'étain à elle seule justifierait largement, d'un point de vue économique, le voyage de ce navire, sans qu'il fût besoin d'y ajouter une cargaison complémentaire d'importance ${ }^{54}$. Par ailleurs, si la présence d'une cargaison complémentaire peut sembler cohérente afin d'optimiser l'espace de chargement, la question de la stabilité du navire vient contrebalancer ce premier aspect. Avec sa seule cargaison d'étain, relativement bien centrée et disposée à fond de cale, le navire devait en effet présenter la meilleure des configurations de navigation : centre de gravité relativement bas et un franc-bord conséquent. En revanche, la présence d'un chargement complémentaire placé au-dessus des lingots ${ }^{55}$ aurait eu pour conséquence directe de réduire à la fois la stabilité transversale de l'embarcation ainsi que son franc-bord, et ainsi d'augmenter le risque de naufrage. Dès lors se pose la question de l'intérêt d'un éventuel profit supplémentaire, économiquement négligeable compte tenu de la valeur des lingots d'étain, mais qui mettrait en revanche en péril l'ensemble de la cargaison.

Le chargement a pu se faire dans un quelconque port du nord de l'Espagne qui avait au préalable réceptionné un ensemble de lingots d'étain, provenant peut-être des mines de l'ouest de la Péninsule. Mais cela nous paraît peu probable. On ne connaît aujourd'hui aucun port suffisamment important entre Èbre et Pyrénées qui ait joué le rôle de port-entrepôt, autrement dit qui ait été un centre de redistribution en Méditerranée occidentale de marchandises de provenances distinctes. Au nord des Pyrénées, c'est en revanche le cas de Narbonne qui reçoit et redistribue des marchandises d'origines différentes : il en va ainsi de tous les types d'amphores et les céramiques fines présentes sur l'épave, en particulier les Oberaden 74 et les Tarraconaise 1, qui sont à l'inverse peu attestées dans d'autres ports importants de la Gaule. Capo Sagro 2 représente également, rappelons-le, la plus importante cargaison connue pour ces amphores à fond plat qui ont connu une large diffusion à travers les voies d'eau. Il en va tout autant du métal, et en particulier du fer de la Montagne Noire (Rico 2011, p. 52-56), ainsi que, peut-être, de l'étain breton, selon l'hypothèse déjà formulée à propos de Port-Vendres II.

Est-ce suffisant pour faire de l'étain de Capo Sagro 2 un étain breton ? Sans doute pas. La typologie n'est d'aucune aide. $\mathrm{Si}$ les lingots sont morphologiquement proches de certains lingots de Bagaud 2 et de Mèdes 1, l'incertitude sur leur provenance subsiste. Toutefois nous pensons qu'une origine hispanique serait la moins probable. Pour l'heure en effet, on ne connaît aucune épave renfermant un chargement de métal hispanique, plomb ou cuivre, dont on pourrait envisager qu'il a été assemblé dans un deuxième temps dans un port-entrepôt comme Narbonne. Les épaves sont homogènes du point de vue des produits, métaux et amphores, qui les constituent, ce qui témoigne d'un commerce direct depuis un port principal comme Hispalis ou Gades.

On émettra donc l'hypothèse que le navire Capo Sagro 2 était parti d'un port du sud de la Gaule, qui a de fortes chances d'être Narbonne, avec une cargaison principale de lingots d'étain d'origine bretonne complétée par quelques dizaines d'amphores vinaires de Tarraconaise alors disponibles dans le même port. L'Italie - et Rome plus particulièrement - où la demande en étain était forte et d'où proviennent une partie des céramiques, des amphores de l'Adriatique, et peut-être la dotation de bord, nous semble la destination la plus vraisemblable de cette cargaison qui a sombré pratiquement au milieu du couloir maritime qui sépare la Corse et la Péninsule, un peu au nord de Bastia et de l'île d'Elbe. L'attestation à Rome-Ostie d'un type d'amphore assez rare ailleurs en Italie comme les Oberaden 74 renforce considérablement cette hypothèse de destination.

Franca CIBECCHINI Ministère de la Culture, DRASSM, Marseille Aix Marseille Univ, CNRS, Minist Culture, CCJ, Aix-en-Provence, France franca.cibecchini@culture.gouv.fr

Christian RICO Université Toulouse Jean-Jaurès, TRACES, UMR 5608 rico@univ-tlse2.fr

Pierre PovedA Aix Marseille Univ, CNRS, CCJ, Aix-en-Provence, France poveda@mmsh.univ-aix.fr
54. On distinguera par là la vingtaine d'amphores Oberaden 74 qui constituent, certes, un chargement complémentaire placé en bordure du chargement de lingots, mais dont l'importance en termes de volume et de poids nous paraît négligeable.
55. La dégradation des qualités nautiques du navire étant liée à la hauteur du centre de gravité de la cargaison et de l'importance de celle-ci, tout dépend donc de la densité et de l'importance de celle-ci : quelques ballots de laines auront une influence mineure sur le comportement de l'embarcation, tandis que plusieurs sacs de blés auront une influence non négligeable. 


\section{BIBLIOGRAPHIE}

Ballard R. D., Stager L., Master D., Yoerger D. Mindell L., Whitcomb L., Singh, H. Piechota D.

2002 Iron Age Shipwrecks in Deep Water off Ashkelon, Israel, AJA 106, p. 151-168.

BERNARD H.

2003 Plage de Losari, Bilan Scientifique du DRASSM 1998, Paris, Ministère de la Culture, p. 59-60.

Bernard H., Domergue Cl.

1991 Les lingots de plomb de l'épave romaine Sud Perduto 2 (Bouches de Bonifacio, Corse), Bulletin de la Société des Sciences Historiques et Naturelles de la Corse, 111, p. 41-96.

BERTI F. (ÉD.)

1990 Fortuna Maris. La nave romana di Comacchio, catalogue de l'exposition, Bologne, Nuova Alfa editoriale.

Brulet R., Vilvorder F., Delage R.

2010 La céramique romaine en Gaule du Nord. Dictionnaire des céramiques. La vaisselle à large diffusion, Turnhout, Brepols.

Carre M.-B., Monsieur P., Pesavento Mattioli S.

2014 Transport Amphorae Lamboglia 2 and Dressel 6A: Italy and/or Dalmatia? Some clarifications, JRA, 27, p. 417-428.

Carreras Monfort C., González Cesteros H.

2012 Ánforas tarraconenses para el limes germano. Una nueva visión sobre las Oberaden 74, dans D. Bernal Casasola, A. Ribera (éd.). Cerámicas Hispanorromanas II, Cadix, p. 207-277.

2013 Las ánforas de los primeros campamentos de Neuss (Renania, Alemania), dans D. Bernal Casasola, M. Bustamante Alvarez, L. C. Juan Tovar (dir.), Hornos, talleres y focos de producción alfarera en Hispania, SECAH I, vol. II, Cadix, San Martín de Valdeiglesias, Universidad de Cádiz, Sociedad de Estudios de la Cerámica Antigua en Hispania, p. 281-298.

\section{Cavalier M.}

1985 Il relitto A (Roghi) del Capo Graziano di Filicudi, dans Archeologia Subacquea 2. Isole Eolie, Rome, Poligrafico e Zecca dello Stato (Suppl. Bollettino d'Arte, 29), p. 101-107.

CERDÀ J.D.

1980 La nave romano-republicana de la Colonia de San Jordi, Ses Salines, Mallorca, Majorque, Cort (Monografías del Museo de Mallorca, 6).

Charlin G., Gassend J.-M., Lequément R.

1978 L'épave antique de la baie de Cavalière (Le Lavandou, Var), Archaeonautica, 2, p. 9-93.

Cibecchini F.

2007 Tonnellaggi e rotte in età republicana. Il contributo dei relitti del Mediterraneo Occidentale, dans J. Pérez Ballester, G. Pascual Berlanga (éd.), Comercio, redistribución y fondeaderos: La navegación a vela en el Mediterráneo, Actas de la V Jornadas Internacionales de Arqueología Subacuática, Gandía, 2006, Valence, Universitat de València, p. 483-499.

2015 Les épaves antiques à grande profondeur en Corse, dans $L a$ Corse et le monde méditerranéen des origines au Moyen-Âge. Échanges et circuits commerciaux, 2, Actes du colloque, Bastia, 2013, Bastia, Société des sciences historiques et naturelles de la Corse (Bulletin de la Société des Sciences Historiques et Naturelles de la Corse, 748-749), p. 7-23.

2016 Et fiat lux... à 20.000 lieux sous la mer ! Nouvelles données sur la cargaison de lampes de l'épave profonde Aléria 1, dans D. Djaoui (dir.), Histoires Matérielles : terre cuite, bois, métal et autres objets, des pots et des potes: Mélanges offerts à Lucien Rivet, Autun, Édition Mergoil, p. 193-201.
Chidiroglou M.

2012 Jugs, one-handled cups, filter jugs, olpai and unguentaria, dans N. Kaltsas, E. Vlachogianni, P. Bouyia (eds), The Antikythera Shipwreck: the ship, the treasures, the mechanism, Exhibition National Archaeological Museum, Athènes, Kapon Editions, p. 196-197.

Colls D., Domergue Cl., Laubenheimer F. Liou B.

1975 Les lingots d'étain de l'épave Port-Vendres II, Gallia, 33, p. 61-94.

Colls D., Étienne R., Lequément R., Liou B., Mayet Fr.,

1977 L'épave Port-Vendres II et le commerce de la Bétique à l'époque de Claude, Paris, éditions du CNRS (Archaeonautica, 1).

Domergue Cl.,

2008 Les mines antiques. La production des métaux aux époques grecque et romaine, Paris, Picard.

Domergue Cl., Rico Chr.

2014 Les itinéraires du commerce du cuivre et du plomb hispaniques à l'époque romaine dans le monde méditerranéen, dans La Corse et le monde méditerranéen des origines au Moyen Âge. Échanges et circuits commerciaux, 1, Actes du colloque, Bastia, 2013, Bastia, Société des sciences historiques et naturelles de la Corse (Bulletin de la Société des Sciences Historiques et Naturelles de la Corse, 746-747), p. 135-168.

À paraître L'approvisionnement en métaux de l'Occident méditerranéen à la fin de la République et sous le Haut-Empire. Flux, routes, organisation, dans International Congress Infrastructure and Distribution in Ancient Economies. The Flow of Money, Goods and Services, Vienna, 2014.

Domergue Cl., Quarati P., Nesta A., Obejero G., Trincherini, P.R.

2012 Les isotopes du plomb et l'identification des lingots de plomb romains des mines de Sierra Morena. Questions de méthode : l'exemple des lingots de l'épave Cabrera 4, Pallas, 90, p. 243-256.

Drap P., Seinturier J., Hijazi B., Merad D., Boi J.-M.,

Chemisky B., Seguin E., Long L.

2015 Le projet ROV 3D : relevé sous-marin en grande profondeur par photogrammétrie. Applications à l'archéologie sous-marine, Revue XYZ, 142, p. 28-40.

EHMIG U.

2010 Dangstetten IV. Die Amphoren: Untersuchungen zur Belieferung einer Militäranlage in augusteicher Zeit und den Grundlagen archäologischer Interpretation von Fund und Befund, Stuttgart, Theiss (Forschungen und Berichte zur Vor- und Frühgeschichte in Baden-Württemberg, 117).

Fontaine S., Cibecchini F.

2014 An Exceptional Example of Maritime Glass Trade: the Deep Wreck Capo Corse 2 (France, Corsica), Journal of Glass Studies, 55, p. 354-357.

ForTi S., PACI G.

2008 Le anfore Lamboglia 2 dal porto romano di Ancona. Notizie preliminari, Rei Cretariae Romanae Fautorum Acta, 40, p. 315-323.

GonZÁlez Cesteros H.,

2015 Hallazgos de productos tarraconenses en la frontera germana. Un mercado secundario, dans V. Martínez Ferreras (éd.), La difusión comercial de las ánforas vinarias de Hispania CiteriorTarraconensis (s. I a.C. - I. d.C.), Oxford, Archeopress, p. 205-220.

González Cesteros H., Carreras Monfort C.

2016 Oberaden 74 (Zone côtière nord-est de Tarraconaise), Amphorae ex Hispania. Géographie de la production et de la consommation, 
http://amphorae.icac.cat/amphora/oberaden-74-tarraconensisnorthern-coastal-area (consulté en ligne le 10/07/2016).

Hulot O.

2017 Épave Bloscon 1. Finistère. Rapport final d'opération, 17 août-2 septembre 2015, Marseille, Drassm.

Klein S., Rico Chr., Layahe Y., von Kaenel H.-M., Domergue CL., BREY G.

2007 Copper ingots from the western Mediterranean Sea: chemical characterization and provenance studies through lead- and copper isotope analyses, JRA, 20, p. 202-221.

LAUBENHEIMER F.

2015 Les circuits d'exportation des vins de Tarraconaise en Gaule, dans V. Martínez Ferreras (éd.) La difusión comercial de las ánforas vinarias de Hispania Citerior-Tarraconensis (s. I a.C. - I. d.C.), Oxford, Archeopress, p. 181-192.

\section{L'Hour M.}

2014 L'exploration de la Lune (1664) : un chantier laboratoire pour l'archéologie des abysses, Cahiers d'Archéologie Subaquatique XXII, p. 149-187.

LONG L.

1985 L'épave antique Bagaud 2, dans VI Congreso Internacional de Arqueología Submarina, Cartagena, 1982, Carthagène, Ministerio de Cultura, p. 93-98

1987 Quelques précisions sur le conditionnement des lingots d'étain de l'épave antique Bagaud 2 (Var), dans Mines et métallurgie en Gaule et dans les provinces voisines, Actes du colloque, Paris, 1986, Paris, Errance (Caesarodunum, 22), p. 149-163.

1998 Lucius Volteilius et l'amphore de 4e type. Découverte d'une amphore atypique dans une épave en baie de Marseille, dans $E l$ vi a l'antiguitat. Economia, producció i comerç al Mediterrani occidental, Actes II Col.loqui internacional d'arqueologia romana, Badalona, 1998, Badalona, Museu de Badalona (Monografies Badalonines, 14), p. 341-349.

2013 Les produits d'origine italique sur les épaves de la côte gauloise et de la Corse (III ${ }^{\mathrm{e}}$ siècle avant J.-C. - I ${ }^{\text {er }}$ siècle après J.-C.), dans G. Olcese (dir.), Immensa Aequora Workshop. Ricerche archeologiche, archeometriche e informatiche per la ricostruzione dell'economia e dei commerci nel bacino occidentale del Mediterraneo (metà IV sec. a.C. - I sec. d.C.), Atti del Convegno, Roma, 2011, Rome, Quasar, p. 419-438.

2015 Bouches-du-Rhône. Au large des Saintes-Maries-de-la-Mer, Bilan Scientifique du DRASSM 2011, Paris, Ministère de la Culture, p. 56-61.

LONG L., DRAP P.

2010 Au large de Marseille, L'épave Port-Miou C, Bilan Scientifique du DRASSM 2008, Paris, Ministère de la Culture, p. 80-81.

LONG L., Drap P., Gantès L.F.,

2002 Premiers résultats archéologiques sur l'épave Grand-Ribaud F (Giens, Var). Quelques éléments nouveaux sur le commerce étrusque en Gaule, vers 500 avant J.-C., Cahiers d'Archéologie Subaquatique, XIV, p. 5-40.

\section{López Mullor A., Martín Menéndez A.}

2008a Las ánforas de la Tarraconense, dans D. Bernal Casasola, A. Ribera i Lacomba (eds.), Cerámicas hispanorromanas: un estado de la cuestión, Universidad de Cadiz, Cadix, p. 689-724.

2008b Tipologia i datació de les àmfores tarraconenses produïdes a Catalunya, dans A. López Mullor, J. Aquilué Abadías (dir.), La producció i el comerç de les àmfores de la Provincia Hispania Tarraconensis : homenatge a Ricard Pascual i Guasch, Barcelone, Museu d'Arqueologia de Catalunya (Monografies del Museu d'Arqueologia de Catalunya, 8), p. 33-94.

Lo Schiavo F.

1986 Un problema insoluto: il relitto di Capo Bellavista, dans Archeologia subacquea 3, Rome, Poligrafico e Zecca dello Stato (Suppl. Bollettino d'Arte, 37-38), p. 135-138.
Mairécolas M., Pailler J.-M.

2010 Sur les « voies de l'étain » dans l'Ancien Occident. Quelques jalons, Pallas, 82, p. I39-I67.

Maggiani A.

1982 Sant Andrea B, dans Archeologia Subacquea 1, Rome, Poligrafico e Zecca dello Stato (Suppl. 4 Bollettino d'Arte, 11), p. $72-78$

Miró J.,

2016 Tarraconense 1 (Zone côtière nord-est de Tarraconaise), dans Amphorae ex Hispania. Geographie de la production et de la consommation, http://amphorae.icac.cat/amphora/tarraconense1-tarraconensis-northern-coastal-area (consulté en ligne le 10/07/2016).

Molina Vidal J.

2013 Commerce et marchés de vin italique dans le sud de l'Hispanie Citérieure (III ${ }^{\mathrm{e}} \mathrm{I}^{\mathrm{er}}$ siècles av. notre ère), dans $\mathrm{F}$. Olmer (éd.), Itinéraires des vins romains en Gaule. III ${ }^{e} I^{e r}$ siècles avant J.-C. Confrontation de faciès, Montpellier, ADAL Éditions, (Monographies d'archéologie méditerranéenne, Hors-série, 5), p. 195-212

Nieto X., Raurich X.

1998 El transport naval de vi de la Tarraconense, dans El vi a l'antiguitat. Economia, producció i comerç al Mediterrani occidental, Actes del II Col.loqui internacional d'arqueologia romana, Badalona, 1998, Badalona, Museu de Badalona (Monografies Badalonines 14), p. 113-137.

Pallecchi S.

2002 Imortaria di produzione centro-italica: corpus dei bolli, Rome, Quasar.

PARKer A.J.

1992 Ancient Shipwrecks of the Mediterranean and the Roman Provinces, Oxford, BAR (BAR Int. Series, 580).

Pérez Ballester J., Pascual Berlanga G.

2004 The Adriatic amphora type L.2 recovered from the environment of Cartagena (Murcia, Spain), dans M. Pasquinucci, T. Weski (eds), Close encounters: sea- and riverborne trade, ports and hinterlands, ship construction and navigation in antiquity, in the Middle Ages and in modern time, Oxford, Archeopress (BAR Int. Series, 1283), p. 27-37.

Pinedo Reyes J., Alonso Campoy D.

2004 El yacimiento submarino de la isla de Escombreras, dans Scombraria, p. 128-151

PoMey P

1982 Le navire romain de la Madrague de Giens, CRAI, 126, 1, p. $133-154$

1985 Perspectives d'avenir pour l'archéologie sous-marine : l'archéologie en eau profonde, dans $2^{e}$ Journées d'étude sur la plongée scientifique, Bulletin de l'Institut Océanographique de Monaco, n. spécial, 4, p. 59-74.

Pomey P., Rieth É.

2005 L'archéologie navale, Paris, Errance.

Ribera A.

2001 Los pecios con cerámicas calenas de barniz negro: problemas y soluciones, dans L. Pedroni (dir.), Ceramica calena a vernice nera. Produzione e diffusione, Naples, Petruzzi editore, p. 296-306

Rico CHR.

2011 Réflexions sur le commerce d'exportation des métaux à l'époque romaine. La logique du stockage, dans J. Arce, B. Goffaux (éds), Horrea d'Hispanie et de la Méditerranée romaine, Madrid, Casa de Velázquez (Collection de la Casa de Velázquez, 125), p. 41-64. 
Rizzo G.

Le anfore. Ostia e i commerci mediterranei, dans C. Panella, G. Rizzo (dir.), Ostia VI. Le Terme del Nuotatore, Rome, L'Erma di Bretschneider (Studi Miscellanei, 38), p. 65-440.

RODEN C.

1985

Montanarchäologische Quellen des ur- und frühgeschichtlichen Zinnerzbergbaus in Europa, Der Anschnitt, 2-3, p. 50-80.

Sabastia A.

à paraître L'épave Mèdes 1 (Porquerolles, $\mathrm{I}^{\mathrm{er}} \mathrm{s}$. BCE) : résultats de la campagne 2016 et premières interprétations, Scientific Report of Port-Cros national Park, 32.

SALVI D.

1992 Cabras (Oristano). Isola di Mal di Ventre. Da Carthago Nova verso i porti del Mediterraneo: il naufragio di un carico di lingotti di piombo, Bollettino d'Archeologia, 16-18, p. 237-248.

SANCHEZ C.

2009a Production et consommation des céramiques communes de la colonie romaine de Narbonne (II ${ }^{\mathrm{e}}$ s. av./III ${ }^{\mathrm{e}}$ s. ap. J.-C.), dans M. Pasqualini (dir.), Les céramiques communes d'Italie et de Narbonnaise : structures de production, typologies et contextes inédits, II s. av. J.-C.-III ${ }^{e}$ s. ap. J.-C., Actes de la table ronde, Naples, 2006, Naples, Centre Jean-Bérard (Collection du Centre Jean-Bérard, 30), p. 471-492.

2009b Narbonne à l'époque tardo-républicaine (II $-I^{e r}$ s. av. n. è.), chronologies, commerce et artisanat céramique, Montpellier, Association de la RAN (RAN suppl., 38).

2015 Les amphores de Tarraconaise dans les contextes narbonnais, dans V. Martínez Ferreras (éd.), La difusión comercial de las ánforas vinarias de Hispania Citerior-Tarraconensis (s. I a. C. -I. d. C.), Oxford, Archeopress, p. 165-180.
Santamaria CL.

1995 L'épave Dramont «E Paris, éditions du CNRS (Archaeonautica, 13).

SCOMBRARIA

2004 Scombraria : la historia oculta bajo el mar: arqueología submarina en Escombreras (Cartagena), catalogue de l'exposition, Cartagena - Murcia 2004, Murcie, Consejería de Educación y Cultura.

SOURISSEAU J.-CHR.

2015 Xlendi, réflexions sur la cargaison de l'épave, 2015 Reports, projet Groplan,

http://www.lsis.org/groplan/papers/groplan_livrableXlendiCargaisonOctobre2015.pdf (consulté en ligne le 10/11/2016).

TAillez P.

1961 Travaux de l'été 1958 sur l'épave du « Titan » à l'île du Levant (Toulon), dans Actes du II Congrès International d'Archéologie Sous-marine, Bordighera, 1958, Bordighera, Institut international d'Études Ligures, p. 175-198.

TCHERnia A.

1969 Informations archéologiques, Gallia, 27-2, p. 465-499.

Trincherini P.R., Domergue Cl., Manteca I., Nesta A., QuARATi P.

2009 The identification of lead ingots from the Roman mines of Cartagena (Murcia, Spain): the role of lead isotope analysis, JRA, 22-1, p. 123-145.

\section{VENy C., CERDÁ D.}

1972 Materiales arqueológicos de dos pecios de la isla de Cabrera (Baleares), Trabajos de Prehistoria, 29, p. 298-328. 